

\title{
Ethnopharmacological and Ethnobotanical study of Medicinal plants in the High Atlas Central, Morocco
}

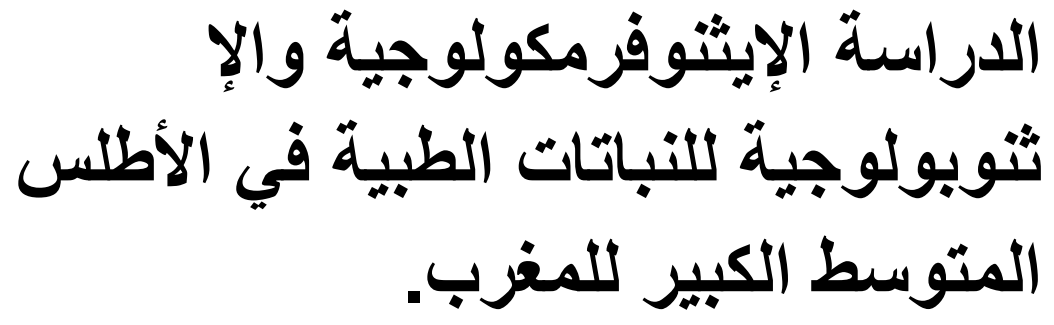

Souad Belhaj, Jamila Dahmani, Nadia Belahbib, Lahcen Zidane

\section{Databases and Inventoiries}

\begin{abstract}
Background: This ethnobotanical and ethnopharmacological study was conducted in the High Atlas Central of Morocco. Its aim is to promote the use of medicinal plants through the realisation of an inventory of these plants and their therapeutic uses in this region.
\end{abstract}

Methods: The ethnopharmacological surveys conducted in the field from 2015 to 2017 have allowed filling 1192 questionnaires. Information was collected by an ethnobotanical and a floristic survey with using open-ended and semi-structured interviews. Data were analyzed using ANOVA and Student's t test; Quantitative ethnobotanical indices such as Fidelity Level (FL), Relative Citation Frequency (RCF) Frequency (F), and Family Importance Value (FIV) were also used to compare data. The medicinal plants were collected, identified and kept at the Biodiversity and Natural Resources Laboratory, Ibn Tofail University, Kenitra.

Results: The study identified a total of 248 medicinal plants used by the local population. They belong to 70 families from which the leading family was Asteraceae, represented by 30 species. Most of these species are used to treat gastrointestinal diseases such as Thymus broussonetii Boiss,
Ceratonia siliqua L. and diabetic diseases such as Anthemis nobilis L., Euphorbia resinifera Berg. These diseases are mainly cured using leaves of the plants cited. The predominant preparation method is decoction.

\section{Correspondence}

Souad Belhaj ${ }^{1^{*}}$, Jamila Dahmani ${ }^{2}$, Nadia Belahbib $^{2}$, Lahcen Zidane ${ }^{1}$

${ }^{1}$ Department of Biology, UFR: Biodiversity and Natural Resources Laboratory, IbnTofail University, PB 133, 14000, Kenitra Faculty of Science, Morocco ${ }^{2}$ Department of Biology, Botanical, Biotechnology and Plant Protection Laboratory, IbnTofail University, PB 13314000, Kenitra Faculty of Science, Morocco

"Corresponding Author:

souadbelhaj2050@gmail.com

Ethnobotany Research \& Applications

20:18 (2020)

Conclusions: This study showed that indigenous people in the High Atlas Central use the plants recorded for the treatment of various diseases. This wealth of plants for therapeutic uses is accompanied by knowledge and practices in phytotherapy acquired by the inhabitants of the High Atlas Central over the centuries. This study keeps a transcribed 
trace of phytotherapy practices. The achieved results are a precious source of ethnomedicinal knowledge that allows the shifting of the researches towards phytochemistry and pharmacology.

Key words: Ethnomedicinal, Therapeutic uses, Medicinal plants, Biodiversity.

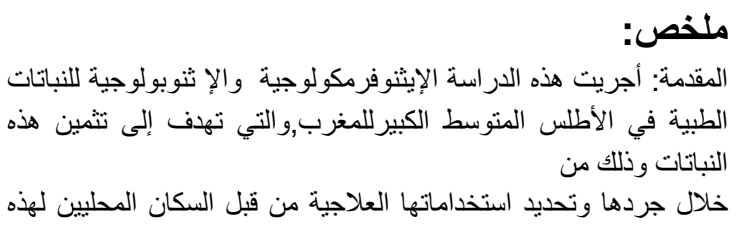
المنطقة .

الأساليب: مكنت هذه الدراسة من ملء 1192 استمارة معلوماتبة تظم دراسات استقصائية إثثوبولوجية ونباتية الجنة أجريت في منطقة البحث, بين

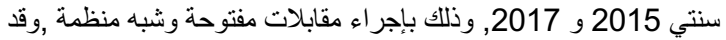

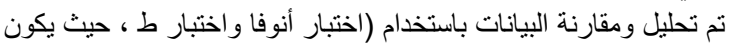

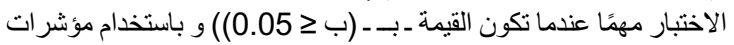

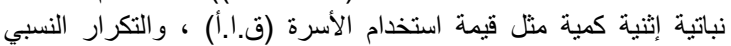

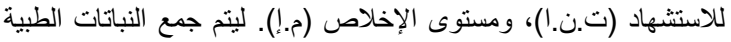

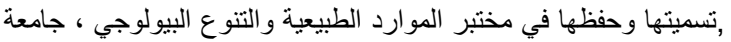
ابن طفيل ، القنيطرة.

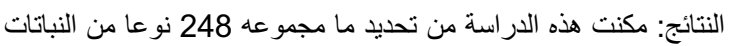



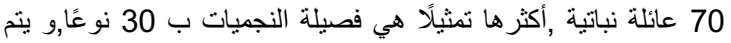

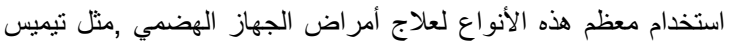

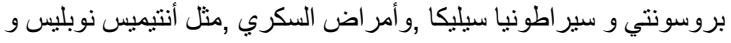

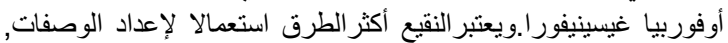
بينما تعد الأوراق الجزء الرئيسي المستخدم في العلاجات.

الاستنتاجات: أظهرت هذه الدر اسة أن السكان المحليين في الأطلس المنوسط



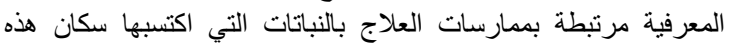

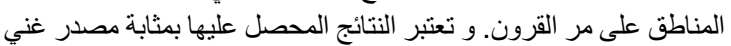

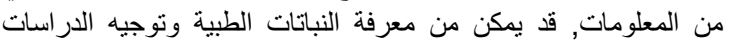
الكيميائية النباتية و الدوائية.

الكلمات المفاتيح: الطب الإثني ، الاستخدامات العلاجية ، النباتات الطبية ، التنوع البيولوجي ؛ المفي:

Introduction

Morocco is a geographical crossroad where several types of bio climates coexist and where the rainfall is irregular, which stimulates the development of a varied flora with a quite high rate of plant endemism within the Mediterranean region (Bellakhdar 2006). Therefore, the natural Moroccan ecosystem contains 4200 vascular plant species. Among these, 382 taxa ( $9 \%$ of the overall flora in Morocco) are exploited for therapeutic, medicinal and aromatic purposes and are classified as aromatic and medicinal plants (Aafi et al. 2005). In Morocco, at least 600 plants are used in phytotherapy (Rejdali 1996), and numerous ethnobotanical studies have been conducted in several regions of Morocco (Bammi \& Douira 2002, Benlamdini et al. 2014, Chaachouay et el. 2019, El
Yahyaoui et al. 2015, Hseini \& Kahhouadji 2007, Salhi et al. 2010).

Besides this promising natural context, Morocco has an ancestral knowledge of herbal medicines, the use of plants for flavoring and preserving some food, as well as the extraction of aromatic principles...

The High Atlas central of Morocco offers a bioclimatic variety, and a distinctly unique geography which is characterized by an altitude gradient (plain, piedmont and mountains) that permits the installation of a rich flora. Despite the richness in plant biodiversity I data about the plants of this region is scarce and fragmentary (El Alami \& Chait 2017, El Alami et al. 2016). Ethnobotany and ethnopharmacology examine indigenous people's knowledge of the use of plants manage or cure diseases. Therefore it is important to keep up a written record of all phytotherapy practices, whose transmission to futures generations, hitherto, is based on oral tradition. We present the results of the floristic study, ethnopharmacological and ethnobotany of medicinal plants used by local populations, as well as the study of the therapeutic diversity, the methods of preparation and use of plants, this work also records the list of all diseases treated.

\section{Materials and Methods}

\section{Study area}

Due to geographical position and its climate, the High Atlas Central of Morocco offers a tremendous ecological and floristic diversity. In addition, the indigenous population of the region has wide traditional phytotherapeutic knowledge. The study was conducted in the center of the atlas chain of Azilal North, Ouarzazate, and Tinghir South, in the High Central (Fig 1).

The communes of Tannant, Wawla, Ait Tamlil, Aït Majden, Aït M'hamed, Zaouiat Ahansal, Tabant, Aït abbas, Aït blal, Sidi-boulkhalef, Tifni, Tidli-Fetouaka, Ouzoud, Aït Bou Oulli, belonging to the city of Azilal which is geographically located in the center of the Kingdom and belongs to the economic region of Beni Mellal-Khenifra, which was born from the 2015 regional division of the three former regions: Meknes-Tafilalet, Chaouia-Ouardigha and TadlaAzilal (Official Gazette, 2015). It covers an area of about 1 million hectares, all mountainous, with the exception of a tiny part of the Tadla plain.

Administratively, the Béni Mellal-Khenifra region comprises five provinces: Azilal, Béni Mellal, Fquih Ben Salah, Khenifra and Khouribga, 135 communes including 16 municipalities and 119 rural Communes (HCP, 2018). 


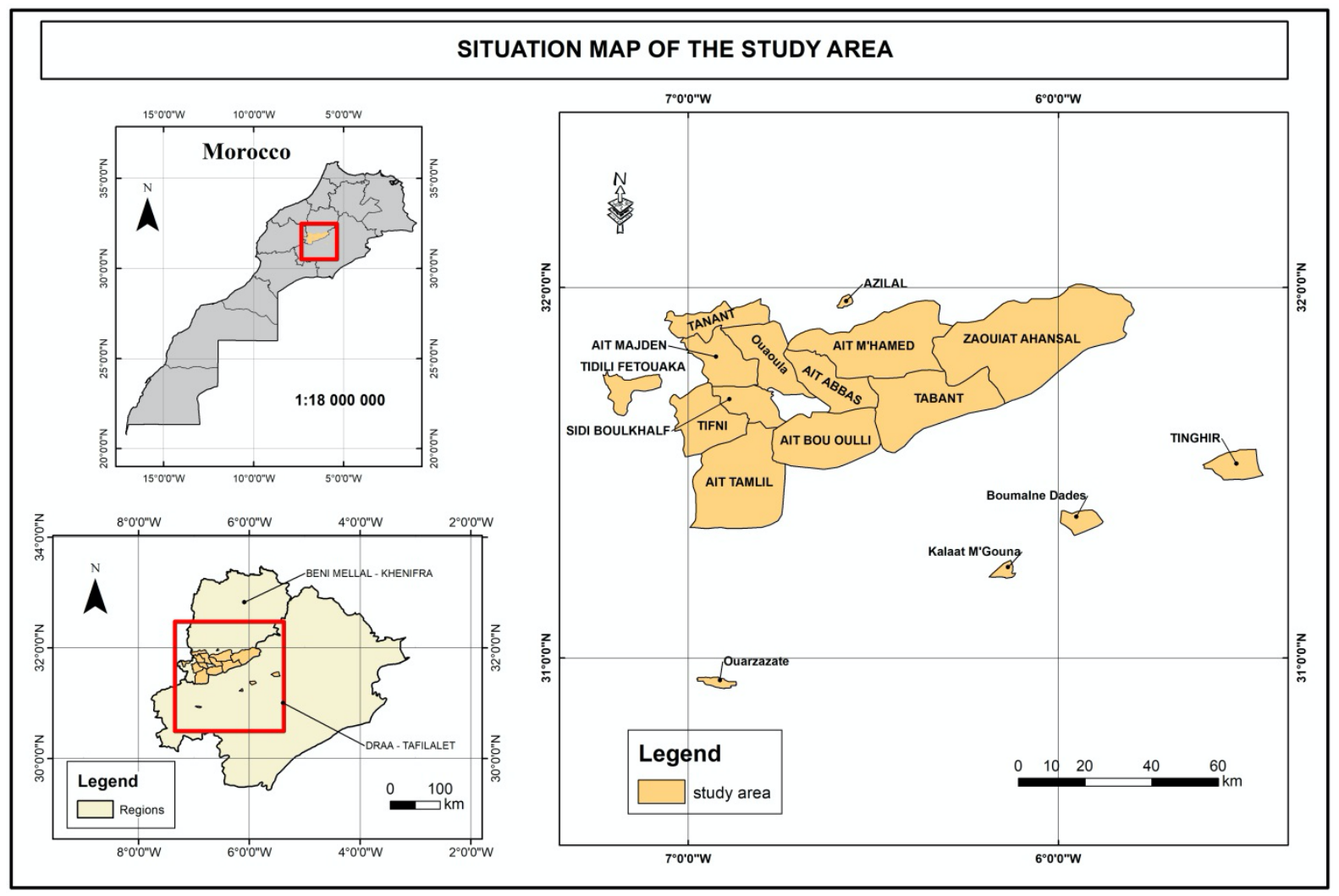

Figure 1:.Map situation of the study area: High Central Atlas (Realized by Belhaj according to the administrative division of 2015, Arcgis 10.3)

About $80 \%$ of the surface area of the Province is located at an altitude of more than $1000 \mathrm{~m}$ and $60 \%$ above $1500 \mathrm{~m}$ (Taibi et al. 1995).

The climate is generally Mediterranean and spreads over the four seasons. It is characterized by abundant rainfall during winter and spring and a very severe summer drought (Sauvage \& Vindt1952, Ouchbani \& Romane1995).

Geologically, the area of the province extends over nearly one million hectares and covers a large part of the high limestone Atlas of the secondary age (Pique 1994). Its population is 2520776 inhabitants, of which 1282037 are rural populations according to the national census of the population 2014 (HCP, 2018), which explains the high illiteracy rate, which is $52 \%$. The main plant formations in the area are as follows: The Holm oaks, which occupy an area of $205,000 \mathrm{Ha}$ or $57.59 \%$ of total forest area of the province followed by Junipers with $18.15 \%$, while Maritime Pine is only represented by $0.29 \%$ and secondary species by $10.46 \%$, although planted forests occupy 9149 ha or $2.61 \%$.
In the southern slope, this work was carried out in the provinces of Tinghir, Ouarzazate belonging to the Draa-Tafilalet region, which was established like the 11 other regions of the Kingdom, in accordance with Decree of 20 February, 2015, and published in Official Bulletin No: 6340 of 05 March, 2015 (Bulletin officiel, 2015). It covers an area of $88,836 \mathrm{~km}$ : With a total area of $1,112,460$ ha, the province of Ouarzazate is bordered to the north by the province of Azilal and Marrakech, to the east by the province of Tinghir, to the south by the province of Tata and Zagora and to the west by the province of Taroudant. It belongs to the arid bioclimatic zone with a continental tendency; the soils of this zone are $75 \%$ clayey-silt soils, with little evolution of alluvial inputs at $20 \%$ and skeletal inputs at $5 \%$ (HCP, 2018).

Special crops in the province occupy small areas but still provide a substantial income for producer. This includes saffron, which occupies 85 hectares with an average annual production of $215 \mathrm{Kg}$ (HCP, 2018). 
-With a total area of 908,960 ha, Tinghir province is bordered to the north by the province of Azilal, to the east by the province of Errachidia, to the south and west by the province of Ouarzazate, it includes 2 circles, namely Tinghir, and Boumalne, 3 municipalities (Tinghir, Kelaa M'gouna and Boumalne Dadés) and 17 rural commons. It belongs to the arid bioclimatic stage with a continental tendency. The Total population of the province of Tinghir is 229666 inhabitants of whom 168,084 (73.19\%) are rural. The soils of the two sub-basins, Todgha and Dades-Mgoun, are in most cases alluvial, undeveloped, deep, silty-sandy and sandy. These soils are $75 \%$ iso humic, $20 \%$ alluvial and $5 \%$ skeletal (HCP, 2018). Special crops in the province occupy small areas but still provide a substantial income to producers, particularly perfume roses, which have produced an average of 2743 tons over the past five years (HCP, 2018).

\section{Data collection tools and procedures}

Twosurveys (ethnopharmacological and floristic) were carried out in the study area usingsemistructured questionnaires, personal inteviews and focus group discussion following the method of Martin (2004).

The field surveys were conducted between 2015 and 2017, by using 1192 questionnaires (Appendix A) administered to 824 residents and sellers of medicinal plants (herbalists and druggists) in the high central atlas of Morocco, the herbalists are a credible source of information, because they have a long expertise regarding the flora and fauna which helps gathering more information on these plants and how they are used.Field trips were also made to observe and collect the plant species, together with information about the vernacular names of the medicinal plants used in the area, types of treated diseases, parts of the plant used, methods of preparation and administration. Standard method was followed with regard to collection of plant materials, drying, mounting, preparation and preservation of plant specimens (Jain 1964). The taxonomic identification of these plants was done in the field and at the Laboratory of Biodiversity and Natural Resources (LBNR) Faculty of Science, Ibn Tofail University, and using available herbaria, directories and flora (Emberger \& Maire, Year, Fennane et al. 1999, Fennane \& Ibn Tattou 2005, Quezel \& Santa 1962, 1963, Sauvage \& Vindt1952). The identified plants were deposited in herbaria and reserved in our laboratory (LBNR).

\section{Data analysis}

The results obtained were analyzed using the descriptive and quantitative statistical method to compare the means between interviewed groups
(ANOVA test and Student $\mathrm{T}$ test, The test is significant when $(P \leq 0.05)$; using the level of fidelity ( $F L)$, Frequency $(F)$, Relative Citation Frequency (RFC) and Family Importance Value (FIV). All statistical analyses were performed using SPSS (version 21) and Microsoft Excel Package 2010.

\section{Fidelity Level (FL)}

Fidelity level (FL) index is used to indicate the plant species more ideal for the treatment of specific ailment (Musa et al. 2011). The FL index was calculated using the formula of Friedman et al. (1986): $F L(\%)=N p / N \times 100$. Where $N p$

Is the number of informants that claim a use of a plant species to treat a particular disease, and $\mathrm{N}$ is the number of informants that use the plants as a medicine to treat any given disease.

\section{Relative Frequency of Citation (RFC) and Frequency (F)}

Relative frequency of citation (RFC) is a quantitative method that demonstrates the relative importance of plant species known locally, was also evaluated according to the formula of Vitalini et al. (2013) and Vijayakumar et al. (2015): RFC = F / N with $(0<$ RFC $<1)$. Where $F$ is the number of informants reporting use of a particular species and $\mathrm{N}$ is the total number of the informants

\section{The Family Importance Value (FIV)}

FIV values show the importance of the plant families. It was calculated by using formula of Molares and Ladio, (2009): FIV = FCfamily / Ns. Where FCfamily $=$ is number of families cited by informants and Ns is the total number of informants.

\section{Results and Discussion}

\section{Socio-demographic characteristic of the participants}

A total of 824 informants, including 427 women and 397 men, were interviewed at the High Atlas Centre in Morocco. Both sexes have a long history of using medicinal plants, but women $(51.82 \%)$ have more knowledge about plant species and their medicinal uses than men $(48.17 \%)$. There was no significant difference between the two sexes. (The Student's T test) $(P=0.375)$ (Table1). This result can be explained by the attachment of women to the traditional component as well as by the ease of transmission of this information between them, and they are concerned with the treatment of themselves and their families. These results are in agreement with those reported in other regions of Morocco (EI alami et al. 2017, Idm'hand et al. 2019, Ziyyat et al. 1997). (Table 1)

On marital status, $65.05 \%$ were married, $17.23 \%$ were single, $11.65 \%$ were widowed and $6.06 \%$ were 
divorced, These results can be explained by the fact that married people take care of their health and that of their children, especially in rural areas, in order to minimize the material expenses of the doctor and the pharmacist, the difference between families status was statistically significant $(P=0.000)$. Other ethnobotanical works have been done in this regard (Benlamdini et al. 2014, El Yahyaoui et al. 2015). (Table 1)

$48.42 \%$ of the partcicipants were over 60 years old, while $24.51 \%$ were between 40 to 60 years old and $20.27 \%$ are aged 20 to 40 years old, however, among the informants the youth showed least interest for using the medicinal plants, they stay come in last position with only $6.79 \%$. The difference between age groups and native informations was significant $(P=0.000)$. The accumulated experience with age is the main source of information locally for use of plants in traditional medicine, but we also noted a loss of medicinal plants information, especially the young people, who have tendency to no longer believe too much in this medicine traditional. This explains that the transmission of this information is currently in danger being it is not always ensured (Anyinam 1995). The results of our research are in conformity with precedent ethnobotanical works that were made in morocco (El hilah et al. 2015, Mehdioui et al. 2007, Tahri et al. 2012). (Table 1)

The majority (51.57\%) of the participants were illiterate $(29.97 \%)$ with a primary level $(12.5 \%)$ have a secondary level, while those in university education rarely use medicinal plants $(5.94 \%)$. As well, there is a significant difference among educational level and indigenous knowledge $(P=0.000)$. These results showed that the more the education level increases, the use of medicinal plants decreases,several studies confirm this result (Bouzid et al. 2017, Laadim et al. 2017, Rhatta et al. 2016). (Table 1)

In our study, it was found that the highest number of interviewees $(54.85 \%)$ had a low socio-economic level, while (25.97\%) were unemployed, $15.41 \%$ had an average level, and only $3.76 \%$ had a slightly higher level. there is a significant difference among income/month and indigenous knowledge ( $P$ $=0.000$ ), and this can be explained by the relative frequency of illiteracy in our study area, as well as the high cost of drugs and the average to low job performance of most respondents in this area. These values are in agreement with those reported in other regions of Morocco (Chaachouay et al. 2019, Douiri et al. 2007). (Table 1).

Table 1. Demographic profile of informants interviewed

\begin{tabular}{|c|c|c|c|c|}
\hline Variables & Catrgories & Total & Percentages (\%) & P-values \\
\hline \multirow[t]{2}{*}{ Gender } & Female & 427 & 51.82 & \multirow[t]{2}{*}{0.375} \\
\hline & Male & 397 & 48.17 & \\
\hline \multirow[t]{4}{*}{ Age groups } & $<20$ years & 56 & 6.79 & \multirow[t]{4}{*}{0.000} \\
\hline & $20-40$ & 167 & 20.27 & \\
\hline & $40-60$ & 202 & 24.5 & \\
\hline & $>60$ years & 399 & 48.42 & \\
\hline \multirow[t]{4}{*}{ Marital status } & Married & 563 & 65.05 & \multirow[t]{4}{*}{0.000} \\
\hline & Single & 142 & 17.23 & \\
\hline & Divorced & 50 & 6.06 & \\
\hline & Widower & 96 & 11.65 & \\
\hline \multirow[t]{4}{*}{ Educational level } & Illiterate & 425 & 51.58 & \multirow[t]{4}{*}{0.000} \\
\hline & Primary & 247 & 29.97 & \\
\hline & Secondary & 103 & 12.5 & \\
\hline & University & 49 & 5.94 & \\
\hline \multirow[t]{4}{*}{ Income/month } & Unemployed & 214 & 25.97 & \multirow[t]{4}{*}{0.000} \\
\hline & $350-1500 \mathrm{DH}$ & 452 & 54.85 & \\
\hline & $1500-5000 \mathrm{DH}$ & 127 & 15.41 & \\
\hline & $>5000 \mathrm{DH}$ & 31 & 3.76 & \\
\hline
\end{tabular}

\section{Floristic Analysis \\ Diversity of Medicinal plant species in the study area}

At the end of the survey, 248 species were identified. These medicinal species belong to 203 genera and 70 botanical families, of which only one family (Equisetaceae) belongs to the Pteridophyta besides three of Gymnosperms, namely Cupressaceae with four species, Pinaceae with two species, and Taxaceae with only one species. The 240 remaining species are part of the Angiosperm branching with a clear dominance of the Dicotyledonous species (217 species) on the monocotyledons (23 species) (Table 2). 


\begin{tabular}{|c|c|c|c|c|c|c|c|c|c|c|c|}
\hline Family & Scientific name & $\begin{array}{l}\text { Voucher } \\
\text { number }\end{array}$ & $\begin{array}{l}\text { Common } \\
\text { name }\end{array}$ & $\begin{array}{l}\text { Vernacular } \\
\text { name }\end{array}$ & Used part & $\begin{array}{l}\text { Mode of } \\
\text { preparation }\end{array}$ & Therapeutic uses & FL & FC & RFC & FIV \\
\hline Aizoaceae & $\begin{array}{l}\text { Carpobrotus } \\
\text { edulis (L.) N. } \\
\text { E.Br }\end{array}$ & (LBNR1) & Griffe de sorcier & Charbabbou & Leaves & Friction & Anti-eczema & 100 & 32 & 0.038 & 0.038 \\
\hline \multirow[t]{3}{*}{ Amaranthaceae } & Beta vulgaris $\mathrm{L}$. & (LBNR2) & $\begin{array}{l}\text { Betterave } \\
\text { sucrière }\end{array}$ & Chemandar & Seeds & Powder & $\begin{array}{l}\text { Against weight loss } \\
\text { (with honey). }\end{array}$ & 86 & 56 & 0.067 & \multirow[t]{3}{*}{0.11} \\
\hline & $\begin{array}{l}\text { Chenopodium } \\
\text { Ambrosioides L. }\end{array}$ & (LBNR3) & Anserine & Mkhinza & $\begin{array}{l}\text { Green part } \\
\text { of the } \\
\text { plant }\end{array}$ & $\begin{array}{l}\text { Infusion } \\
\text { Maceration }\end{array}$ & $\begin{array}{l}\text { Antimigraine. } \\
\text { Stomachic. }\end{array}$ & 100 & 146 & 0.177 & \\
\hline & $\begin{array}{l}\text { Spinacia } \\
\text { oleracea L. }\end{array}$ & (LBNR4) & Epinard & Sabanikh & $\begin{array}{l}\text { Aerial part } \\
\text { of the } \\
\text { plant }\end{array}$ & Cooked & Stomachic & 91.5 & 71 & 0.086 & \\
\hline \multirow[t]{2}{*}{ Amaryllidaceae } & Allium cepa L. & (LBNR5) & Oignon & $\begin{array}{l}\text { Lbassala/ } \\
\text { Azalim }\end{array}$ & $\begin{array}{l}\text { The whole } \\
\text { of Onion }\end{array}$ & $\begin{array}{l}\text { Poultice } \\
\text { Cooked } \\
\text { Juice } \\
\text { Raw }\end{array}$ & $\begin{array}{l}\text { Ear-pain. } \\
\text { Anti-ulcer. } \\
\text { Hair care. } \\
\text { Dermatological } \\
\text { affections } \\
\text { Antidiabetic. }\end{array}$ & 96.2 & 85 & 0.103 & \multirow[t]{2}{*}{0.149} \\
\hline & $\begin{array}{l}\text { Allium sativum } \\
\text { L. }\end{array}$ & (LBNR6) & Ail & $\begin{array}{l}\text { Touma/ } \\
\text { Tishert }\end{array}$ & Bulbs & $\begin{array}{l}\text { Suppository } \\
\text { Cooked } \\
\text { Raw } \\
\text { Friction } \\
\text { Massag } \\
\text { Others }\end{array}$ & $\begin{array}{l}\text { Anti- hemeroide. } \\
\text { Antidiabetic. } \\
\text { Lowers blood- } \\
\text { pressure. } \\
\text { Decrease respiratory } \\
\text { affections. } \\
\text { Tooth ache. } \\
\text { Hair care. }\end{array}$ & 87.4 & 161 & 0.195 & \\
\hline \multirow[t]{2}{*}{ Anacardiaceae } & $\begin{array}{l}\text { Pistacia atlantica } \\
\text { Desf. }\end{array}$ & (LBNR7) & $\begin{array}{l}\text { Pistachier de } \\
\text { l'Atlas }\end{array}$ & Lbtam. & $\begin{array}{l}\text { Leaves } \\
\text { Barks } \\
\text { Fruits }\end{array}$ & $\begin{array}{l}\text { Decoction } \\
\text { Inhalation } \\
\text { Powder } \\
\text { Others }\end{array}$ & $\begin{array}{l}\text { Calming ventral. } \\
\text { Stomachic. } \\
\text { Heals kidney } \\
\text { diseases. } \\
\text { Antiseptic }\end{array}$ & 80.16 & 103 & 0.125 & \multirow[t]{2}{*}{0.125} \\
\hline & $\begin{array}{l}\text { Pistacia } \\
\text { lentiscus L. }\end{array}$ & (LBNR8) & Lentisque & Drou & $\begin{array}{l}\text { Leaves } \\
\text { Barks } \\
\text { Roots }\end{array}$ & $\begin{array}{l}\text { Decoction } \\
\text { Infusion } \\
\text { Poultice }\end{array}$ & $\begin{array}{l}\text { Anti-ulcer. } \\
\text { Lowers blood- } \\
\text { pressure } \\
\text { Antidiabetic. } \\
\text { Anti-diarrhea. } \\
\text { Pressure tooth ache } \\
\text { and gum. } \\
\text { Anti-migraine }\end{array}$ & 53.4 & 122 & 0.148 & \\
\hline
\end{tabular}




\begin{tabular}{|c|c|c|c|c|c|c|c|c|c|c|c|}
\hline & $\begin{array}{l}\text { Rhus } \\
\text { pentaphylla } \\
\text { (Jacq.) Desf. }\end{array}$ & (LBNR9) & Sumac vernis & $\begin{array}{l}\text { Tazart/ âzâd, } \\
\text { tazâd }\end{array}$ & $\begin{array}{l}\text { Leaves } \\
\text { Fruits }\end{array}$ & $\begin{array}{l}\text { Decoction } \\
\text { Infusion }\end{array}$ & $\begin{array}{l}\text { Gastro-intestinal } \\
\text { disorders. } \\
\text { Anti-diarrhea. }\end{array}$ & 100 & 86 & 0.104 & \\
\hline \multirow[t]{11}{*}{ Apiaceae } & Ammi majus L. & (LBNR10) & Ammi commun & Tabllawt & $\begin{array}{l}\text { Seeds } \\
\text { Roots } \\
\text { Leaves }\end{array}$ & $\begin{array}{l}\text { Maceration } \\
\text { Poultice } \\
\text { Decoction }\end{array}$ & $\begin{array}{l}\text { Bucco infections. } \\
\text { Emollient. } \\
\text { Against intestinal } \\
\text { pains. }\end{array}$ & 89.6 & 131 & 0.158 & \multirow{11}{*}{0.107} \\
\hline & $\begin{array}{l}\text { Ammi visnaga } \\
\text { (L.) Lam. }\end{array}$ & (LBNR11) & Ammi visnage & Bechnikha & $\begin{array}{l}\text { Seeds } \\
\text { Fruits }\end{array}$ & Decoction & Antidiabetic. & 100 & 62 & 0.075 & \\
\hline & $\begin{array}{l}\text { Ammodaucus } \\
\text { leucotrichus } \\
\text { Coss \& Dur. }\end{array}$ & (LBNR12) & $\begin{array}{l}\text { Cumin } \\
\text { Laineux }\end{array}$ & Kammûn sofi & Fruits & Infusion & $\begin{array}{l}\text { Stomachic. } \\
\text { Calming belly pain } \\
\text { (mixed withthymus } \\
\text { satureioides). }\end{array}$ & 69.2 & 136 & 0.165 & \\
\hline & $\begin{array}{l}\text { Anethum } \\
\text { Graveolens L. }\end{array}$ & (LBNR13) & Aneth & Chibt & Seeds & Decoction & $\begin{array}{l}\text { Stomachic. } \\
\text { Diuretic }\end{array}$ & 83.8 & 68 & 0.082 & \\
\hline & $\begin{array}{l}\text { Angelica } \\
\text { Archangelica L. }\end{array}$ & (LBNR14) & $\begin{array}{l}\text { Angélique } \\
\text { officinale }\end{array}$ & $\begin{array}{l}\text { Hachichat } \\
\text { Malaeka }\end{array}$ & $\begin{array}{l}\text { Stems } \\
\text { Seeds }\end{array}$ & $\begin{array}{l}\text { Infusion } \\
\text { Maceration }\end{array}$ & $\begin{array}{l}\text { Treat the intestinal } \\
\text { spasms. } \\
\text { Stomachic. } \\
\text { Sedative. }\end{array}$ & 81.8 & 99 & 0.120 & \\
\hline & $\begin{array}{l}\text { Apium } \\
\text { graveolens L. }\end{array}$ & (LBNR15) & Céleri & Krafes & $\begin{array}{l}\text { Leaves } \\
\text { Leafy }\end{array}$ & $\begin{array}{l}\text { Decoction } \\
\text { Infusion }\end{array}$ & $\begin{array}{l}\text { Diuretic. } \\
\text { Antirheumatic. }\end{array}$ & 95.2 & 76 & 0.092 & \\
\hline & $\begin{array}{l}\text { Anethum } \\
\text { graveolens L. }\end{array}$ & (LBNR16) & Aneth & Tabech & Flowers & Decoction & $\begin{array}{l}\text { Calming stomach } \\
\text { ache. }\end{array}$ & 100 & 88 & 0.106 & \\
\hline & Carum carvi L. & (LBNR17) & Carvi & Karwia & Seeds & $\begin{array}{l}\text { Infusion } \\
\text { Powder }\end{array}$ & $\begin{array}{l}\text { Antidiabetic. } \\
\text { Antirheumatic. } \\
\text { Carminative }\end{array}$ & 84.09 & 122 & 0.148 & \\
\hline & $\begin{array}{l}\text { Coriandrum } \\
\text { sativum } \mathrm{L} \text {. }\end{array}$ & (LBNR18) & Coriandre & Kezbour & $\begin{array}{l}\text { Seeds } \\
\text { Leaves } \\
\text { Aerial } \\
\text { parts }\end{array}$ & $\begin{array}{l}\text { Infusion } \\
\text { Poultice } \\
\text { Decoction } \\
\text { Powder } \\
\text { Friction }\end{array}$ & $\begin{array}{l}\text { Stomachic. } \\
\text { Antidiabetic } \\
\text { Anti-diarrhea. } \\
\text { Diuretic. } \\
\text { Antirheumatic. }\end{array}$ & 93.8 & 95 & 0.115 & \\
\hline & $\begin{array}{l}\text { Daucus carota } \\
\text { L. }\end{array}$ & (LBNR19) & Carotte & Khizzo & $\begin{array}{l}\text { Whole } \\
\text { plant }\end{array}$ & $\begin{array}{l}\text { Juice } \\
\text { Infusion }\end{array}$ & $\begin{array}{l}\text { Calms intestinal and } \\
\text { urinary } \\
\text { inflammations. } \\
\text { Stimulates the blood } \\
\text { circulation }\end{array}$ & 94 & 61 & 0.074 & \\
\hline & $\begin{array}{l}\text { Eryngium } \\
\text { ilicifolium Lamk.. }\end{array}$ & (LBNR20) & Panicaut & Zerriga & $\begin{array}{l}\text { Whole } \\
\text { plant }\end{array}$ & Powder & $\begin{array}{l}\text { Heals angina (mixed } \\
\text { with honey) }\end{array}$ & 100 & 54 & 0.065 & \\
\hline
\end{tabular}




\begin{tabular}{|c|c|c|c|c|c|c|c|c|c|c|c|}
\hline & $\begin{array}{l}\text { Foeniculum } \\
\text { vulgare Mill. }\end{array}$ & (LBNR21) & Fenouil & Nafae & $\begin{array}{r}\text { Whole } \\
\text { plant }\end{array}$ & $\begin{array}{l}\text { Poultice } \\
\text { Decoction } \\
\text { Infusion }\end{array}$ & $\begin{array}{l}\text { Aperitif. } \\
\text { Laxative. } \\
\text { Antidiabetic. } \\
\text { Antiseptic. } \\
\text { Antirheumatic. } \\
\text { Diuretic. } \\
\text { Against bowel pains. }\end{array}$ & 86.3 & 92 & 0.111 & \\
\hline & $\begin{array}{l}\text { Ferula } \\
\text { communis L. }\end{array}$ & (LBNR22) & Faux fenouil & Boubal & Fruits & Cooked & $\begin{array}{l}\text { Improves blood } \\
\text { circulation. }\end{array}$ & 100 & 38 & 0.046 & \\
\hline & $\begin{array}{l}\text { Petroselinum } \\
\text { sativum } \\
\text { Hoffman. }\end{array}$ & (LBNR23) & Persil & Maadnouss & $\begin{array}{l}\text { Leafy } \\
\text { stems }\end{array}$ & $\begin{array}{l}\text { Decoction } \\
\text { Infusion }\end{array}$ & $\begin{array}{l}\text { Soothes the urinary } \\
\text { pains. } \\
\text { Against kidney } \\
\text { stones. } \\
\text { Emmenagogue }\end{array}$ & 85.8 & 116 & 0.140 & \\
\hline & $\begin{array}{l}\text { Pimpinella } \\
\text { anisum L. }\end{array}$ & (LBNR24) & Anis vert & Habthlawa & Seeds & Decoction & $\begin{array}{l}\text { Sedative. } \\
\text { Antidiabetic. }\end{array}$ & 91.1 & 102 & 0.123 & \\
\hline & $\begin{array}{l}\text { Ridolfia segetum } \\
\text { Moris }\end{array}$ & (LBNR25) & $\begin{array}{l}\text { Aneth des } \\
\text { moissons }\end{array}$ & Tebche & Seeds & $\begin{array}{l}\text { Decoction } \\
\text { Powder }\end{array}$ & $\begin{array}{l}\text { Stomachic } \\
\text { Antidiabetic. }\end{array}$ & 94.4 & 71 & 0.086 & \\
\hline & $\begin{array}{l}\text { Smyrnium } \\
\text { Olusatrum L. }\end{array}$ & (LBNR26) & Maceron & Lhayar & $\begin{array}{l}\text { Young } \\
\text { shoots } \\
\text { Leaves }\end{array}$ & $\begin{array}{l}\text { Cooked } \\
\text { Powder }\end{array}$ & $\begin{array}{l}\text { Emmenagogue. } \\
\text { Soothes and stops } \\
\text { asthma attacks. } \\
\text { Heals wounds }\end{array}$ & 86.7 & 67 & 0.081 & \\
\hline & $\begin{array}{l}\text { Thapsia } \\
\text { garganica L. }\end{array}$ & (LBNR27) & Faux fenouil & Addryas & $\begin{array}{l}\text { Leaves, } \\
\text { Roots }\end{array}$ & Poultice & $\begin{array}{l}\text { Antirheumatic (with } \\
\text { honey). } \\
\text { Heals the hair. }\end{array}$ & 89 & 119 & 0.144 & \\
\hline \multirow[t]{2}{*}{ Apocynaceae } & $\begin{array}{l}\text { Caralluma } \\
\text { europaea } \\
\text { (Guss.) N.E.Br. }\end{array}$ & (LBNR28) & Caralluma & Darmouss & $\begin{array}{l}\text { Snowshoe } \\
\mathrm{s}\end{array}$ & $\begin{array}{l}\text { Decoction } \\
\text { Juice } \\
\text { Powder }\end{array}$ & $\begin{array}{l}\text { Antidiabetic. } \\
\text { Aperitif. } \\
\text { Against cough and } \\
\text { asthma } \\
\text { Treats cysts of the } \\
\text { genital tract } \\
\text { (associated with } \\
\text { honey) }\end{array}$ & 68.7 & 112 & 0.135 & \multirow[t]{2}{*}{0.111} \\
\hline & $\begin{array}{l}\text { Nerium oleander } \\
\text { L. }\end{array}$ & (LBNR29) & Laurier rose & Ddefla & $\begin{array}{l}\text { Leafy } \\
\text { stems }\end{array}$ & $\begin{array}{l}\text { Infusion } \\
\text { Poultice } \\
\text { Fumigation } \\
\text { Others }\end{array}$ & $\begin{array}{l}\text { Reduce dandruff. } \\
\text { Treat eczema } \\
\text { especially feet. } \\
\text { Treat leprosy } \\
\text { Antidiabetic }\end{array}$ & 96.9 & 72 & 0.087 & \\
\hline
\end{tabular}




\begin{tabular}{|c|c|c|c|c|c|c|c|c|c|c|c|}
\hline Araliaceae & Hedera helix L. & (LBNR30) & Lierre rampant & Lwwaya & Leaves & $\begin{array}{l}\text { Infusion } \\
\text { Poultice } \\
\text { Decoction }\end{array}$ & $\begin{array}{l}\text { Respiratory tract } \\
\text { treatment } \\
\text { Anti cellulite. } \\
\text { Antirheumatic. } \\
\text { Antiulcer. } \\
\text { Against toothache } \\
\text { and ears. }\end{array}$ & 64 & 79 & 0.095 & 0.095 \\
\hline \multirow[t]{2}{*}{ Arecaceae } & $\begin{array}{l}\text { Chamaerops } \\
\text { humilis L. }\end{array}$ & (LBNR31) & Palmier nain & Ddoum & $\begin{array}{l}\text { Roots } \\
\text { Fruits }\end{array}$ & $\begin{array}{l}\text { Cooked } \\
\text { Raw }\end{array}$ & $\begin{array}{l}\text { Antidiabetic. } \\
\text { Stomachic. }\end{array}$ & 89 & 48 & 0.058 & \multirow[b]{2}{*}{0.061} \\
\hline & $\begin{array}{l}\text { Phoenix } \\
\text { dactylifera L. }\end{array}$ & (LBNR32) & Palmier dattier & Tmar & Fruits & $\begin{array}{l}\text { Raw } \\
\text { Decoction } \\
\text { Cooked }\end{array}$ & $\begin{array}{l}\text { Antidiarrhea. } \\
\text { Antidiabetic } \\
\text { Lowers blood- } \\
\text { pressure } \\
\text { Stomachic. }\end{array}$ & 82.6 & 54 & 0.065 & \\
\hline Aristolochiaceae & $\begin{array}{l}\text { Aristolochia } \\
\text { baetica L. }\end{array}$ & (LBNR33) & $\begin{array}{l}\text { Aristoloche } \\
\text { climatite }\end{array}$ & Brztam & $\begin{array}{l}\text { Leafy } \\
\text { stems }\end{array}$ & $\begin{array}{l}\text { Infusion } \\
\text { Poultice }\end{array}$ & $\begin{array}{l}\text { Stomachic. } \\
\text { Antiseptic. } \\
\text { Against bronchial } \\
\text { inflammation for } \\
\text { children. }\end{array}$ & 74.8 & 31 & 0.037 & 0.037 \\
\hline \multirow[t]{3}{*}{ Aspargaceae } & $\begin{array}{l}\text { Agave } \\
\text { americana L. }\end{array}$ & (LBNR34) & Agave & Sabra & $\begin{array}{l}\text { Leaves } \\
\text { Seve } \\
\text { Roots }\end{array}$ & $\begin{array}{l}\text { Poultice } \\
\text { Cream } \\
\text { Decoction }\end{array}$ & $\begin{array}{l}\text { Antirheumatic. } \\
\text { Softens the hair. } \\
\text { Heal syphilis (with } \\
\text { honey). }\end{array}$ & 96.2 & 32 & 0.038 & \multirow[t]{3}{*}{0.05} \\
\hline & $\begin{array}{l}\text { Asparagus albus } \\
\text { L. }\end{array}$ & (LBNR35) & $\begin{array}{l}\text { Asperge à tiges } \\
\text { blanches }\end{array}$ & Skkoum & $\begin{array}{l}\text { Leaves } \\
\text { Stems } \\
\text { Roots }\end{array}$ & Decoction & $\begin{array}{l}\text { Against diseases of } \\
\text { the kidneys. } \\
\text { Antirheumatic. } \\
\text { Diuretic. }\end{array}$ & 46 & 69 & 0.083 & \\
\hline & $\begin{array}{l}\text { Drimia maritima } \\
\text { (L.) Stearn }\end{array}$ & (LBNR36) & Scille maritime & Basal eddib & Bulbs & Grinding & Heals eye pain. & 92.6 & 25 & 0.03 & \\
\hline \multirow[t]{4}{*}{ Asteraceae } & $\begin{array}{l}\text { Achillea } \\
\text { millefolium } \\
\text { L. }\end{array}$ & (LBNR37) & $\begin{array}{l}\text { Achillée } \\
\text { millefeuille }\end{array}$ & Khala & $\begin{array}{l}\text { Aerial } \\
\text { parts }\end{array}$ & $\begin{array}{l}\text { Infusion } \\
\text { Poultice }\end{array}$ & $\begin{array}{l}\text { Aperitif. } \\
\text { Stomachic. } \\
\text { Emmenagogue. } \\
\text { Lowers blood- } \\
\text { pressure. }\end{array}$ & 58.4 & 67 & 0.081 & \\
\hline & $\begin{array}{l}\text { Achillea } \\
\text { Lipiophylla } \\
\text { M.Bieb. }\end{array}$ & (LBNR38) & Achille & El-qorte & Leaves & $\begin{array}{l}\text { Decoction } \\
\text { Infusion }\end{array}$ & $\begin{array}{l}\text { Antidiabetic. } \\
\text { Stomachic. }\end{array}$ & 98.2 & 81 & 0.098 & \\
\hline & $\begin{array}{l}\text { Achillea } \\
\text { Santolinoides L. }\end{array}$ & (LBNR39) & Achille & Chouihiya & Capitulum & Infusion & $\begin{array}{l}\text { Antidiabetic. } \\
\text { Stomachic. }\end{array}$ & 100 & 43 & 0.052 & \\
\hline & $\begin{array}{l}\text { Anacyclus } \\
\text { pyrethrum (L.) } \\
\text { Link }\end{array}$ & (LBNR40) & $\begin{array}{l}\text { Pyrèthre } \\
\text { d'afrique. }\end{array}$ & Tiguentest & $\begin{array}{l}\text { Whole } \\
\text { plant }\end{array}$ & $\begin{array}{l}\text { Decoction } \\
\text { Infusion }\end{array}$ & $\begin{array}{l}\text { Treat the } \\
\text { toothaches. }\end{array}$ & 89 & 63 & 0.076 & \\
\hline
\end{tabular}




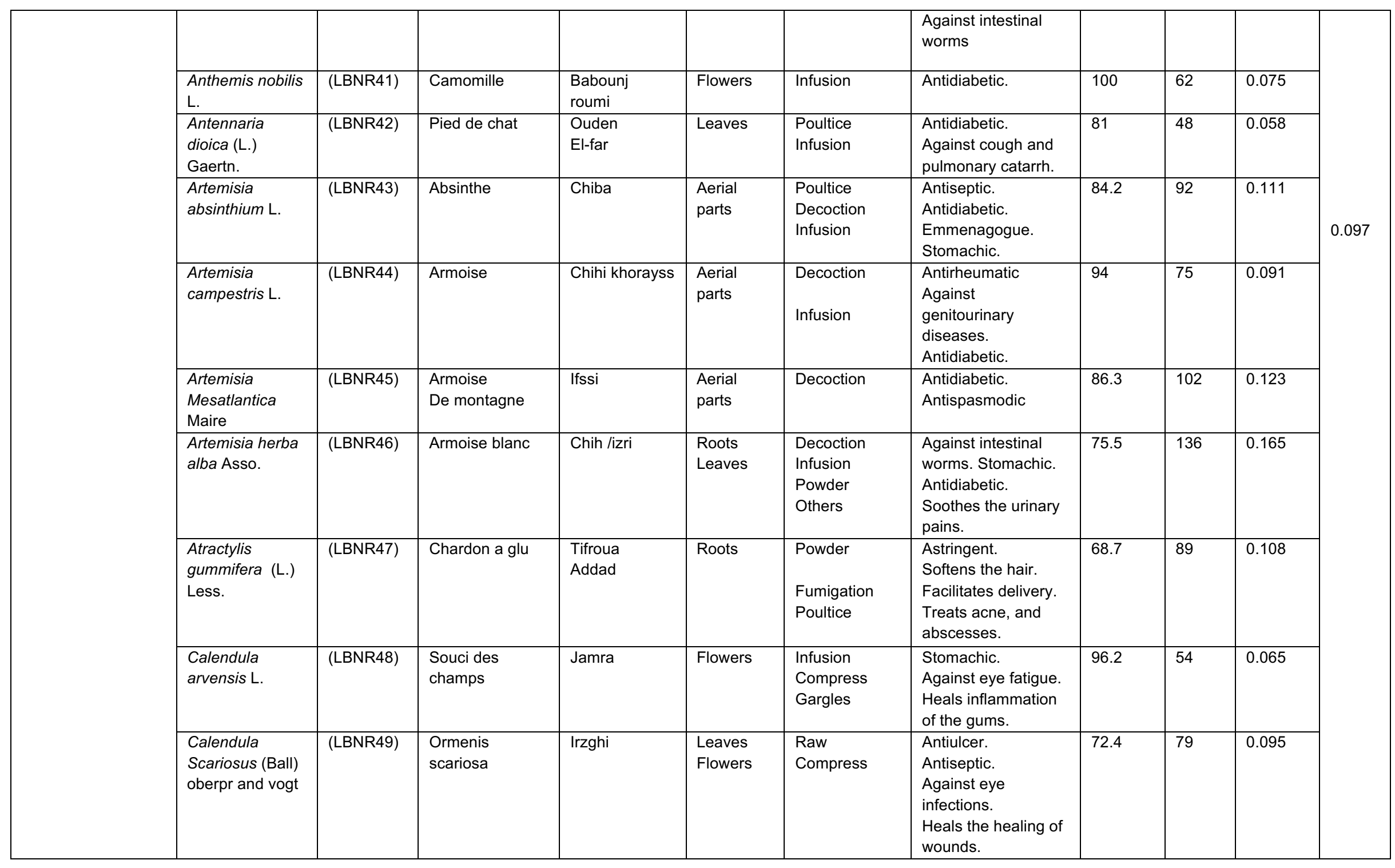




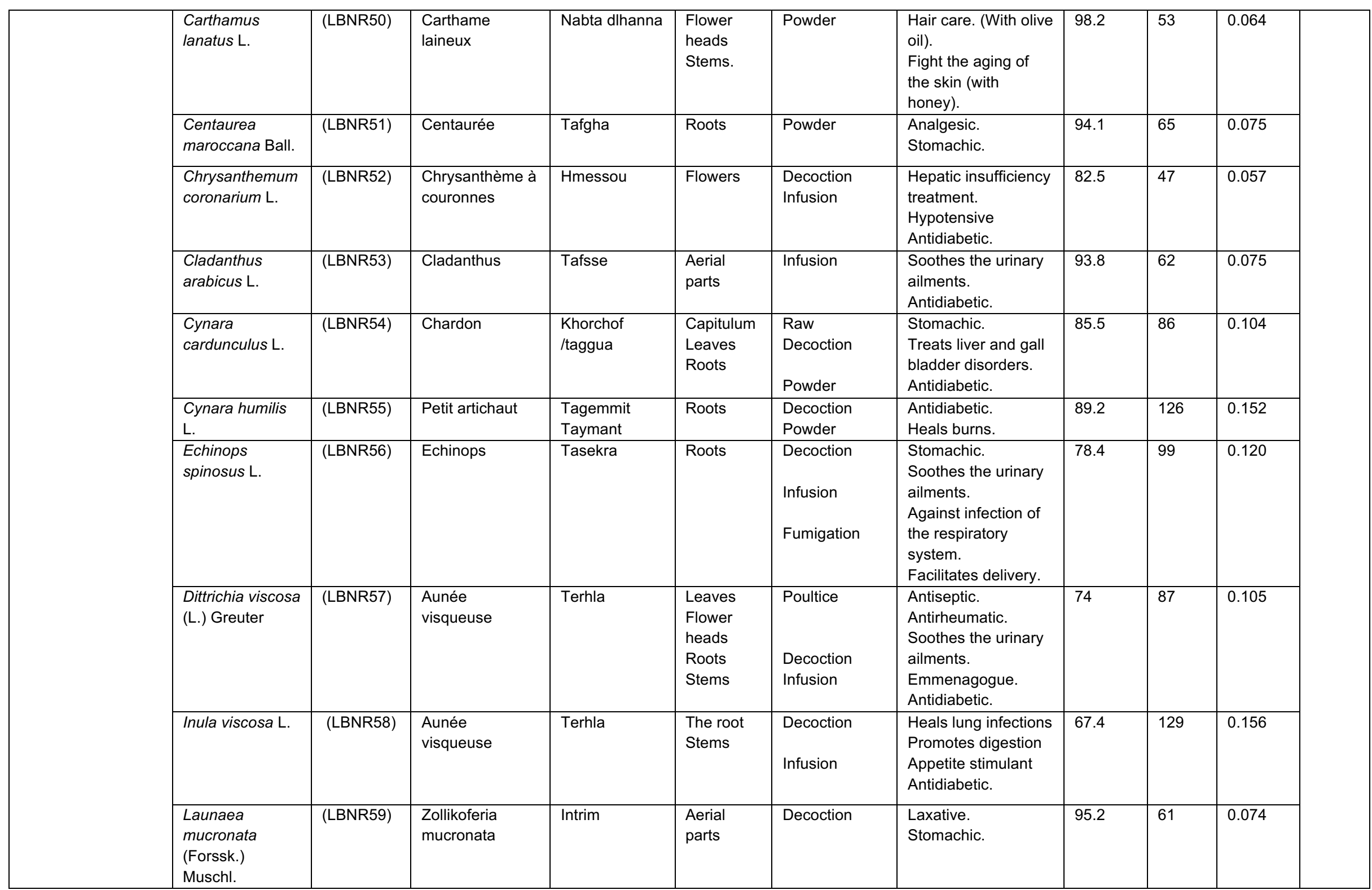




\begin{tabular}{|c|c|c|c|c|c|c|c|c|c|c|c|}
\hline & $\begin{array}{l}\text { Mantisalca } \\
\text { salmantica } \\
\text { (L.) Briq. \& } \\
\text { Cavill. }\end{array}$ & (LBNR60) & $\begin{array}{l}\text { Centaurée de } \\
\text { Salamanque }\end{array}$ & Thazmourth & Leaves & Infusion & $\begin{array}{l}\text { Reduces } \\
\text { gastrointestinal } \\
\text { disorders. }\end{array}$ & 100 & 81 & 0.098 & \\
\hline & $\begin{array}{l}\text { Matricaria } \\
\text { chamomilla L. }\end{array}$ & (LBNR61) & La camomille & Lbabounj & $\begin{array}{l}\text { Rounded } \\
\text { flowers }\end{array}$ & $\begin{array}{l}\text { Decoction } \\
\text { Infusion }\end{array}$ & $\begin{array}{l}\text { Against the pain of } \\
\text { the rules. } \\
\text { Antidiabetic. } \\
\text { Heals bowel pains in } \\
\text { infants. }\end{array}$ & 74.8 & 113 & 0.137 & \\
\hline & $\begin{array}{l}\text { Onopordum } \\
\text { acaulon L. }\end{array}$ & (LBNR62) & $\begin{array}{l}\text { Onopordon à } \\
\text { feuilles } \\
\text { d'acanthe }\end{array}$ & Addad & Roots & Decoction & $\begin{array}{l}\text { Antianemic. } \\
\text { Aperitif. }\end{array}$ & 92.6 & 93 & 0.112 & \\
\hline & $\begin{array}{l}\text { Pallenis spinosa } \\
\text { (L.) Cass. }\end{array}$ & (LBNR63) & Pallénis épineux & Nouged & $\begin{array}{l}\text { Aerial } \\
\text { parts }\end{array}$ & $\begin{array}{l}\text { Decoction } \\
\text { Infusion }\end{array}$ & $\begin{array}{l}\text { Stomachic. } \\
\text { Antidiabetic. }\end{array}$ & 86.9 & 131 & 0.158 & \\
\hline & $\begin{array}{l}\text { Picris } \\
\text { coronopifolia } \\
\text { (Desf.) DC. }\end{array}$ & (LBNR64) & Picride & Lhaydwan & $\begin{array}{l}\text { Whole } \\
\text { plant }\end{array}$ & Powder & Stomachic & 100 & 88 & 0.106 & \\
\hline & $\begin{array}{l}\text { Scolymus } \\
\text { hispanicus L. }\end{array}$ & (LBNR65) & $\begin{array}{l}\text { Scolyme } \\
\text { d'Espagne }\end{array}$ & Taghdut & $\begin{array}{l}\text { Roots } \\
\text { Young } \\
\text { shoots } \\
\end{array}$ & $\begin{array}{l}\text { Decoction } \\
\text { Cooked }\end{array}$ & $\begin{array}{l}\text { Antidiabetic. } \\
\text { Antiulcer. }\end{array}$ & 93.8 & 102 & 0.123 & \\
\hline & $\begin{array}{l}\text { Sonchus } \\
\text { oleraceus } \mathrm{L} .\end{array}$ & (LBNR66) & $\begin{array}{l}\text { laiteron } \\
\text { maraîcher }\end{array}$ & Tifaf & Leaves & Decoction & Antidiabetic. & 100 & 75 & 0.091 & \\
\hline Berbéridaceae & $\begin{array}{l}\text { Berberis } \\
\text { hispanica Boiss } \\
\text { \& Reut }\end{array}$ & (LBNR67) & Les berbéris & Argîs/ izzirki & $\begin{array}{l}\text { Leaves } \\
\text { Fruits }\end{array}$ & $\begin{array}{l}\text { Infusion } \\
\text { Decoction }\end{array}$ & $\begin{array}{l}\text { Heal gastrointestinal } \\
\text { and hepatic atony. } \\
\text { Antidepressant } \\
\text { Antidiabetic. }\end{array}$ & 75.5 & 41 & 0.049 & 0.049 \\
\hline \multirow[t]{2}{*}{ Boraginaceae } & $\begin{array}{l}\text { Borago } \\
\text { officinalis } \mathrm{L} \text {. }\end{array}$ & (LBNR68) & La bourrache & Lsan al ard & $\begin{array}{l}\text { Whole } \\
\text { plant }\end{array}$ & $\begin{array}{l}\text { Raw } \\
\text { Poultice }\end{array}$ & $\begin{array}{l}\text { Antirheumatic. } \\
\text { Calm rashes. }\end{array}$ & 81 & 52 & 0.063 & \multirow[b]{2}{*}{0.056} \\
\hline & $\begin{array}{l}\text { Echium } \\
\text { plantagineum L. }\end{array}$ & (LBNR69) & $\begin{array}{l}\text { Vipérine } \\
\text { annuelle }\end{array}$ & Awnnass & Seeds & Powder & $\begin{array}{l}\text { Against snake bites. } \\
\text { (with honey) }\end{array}$ & 100 & 41 & 0.049 & \\
\hline \multirow[t]{2}{*}{ Brassicaceae } & $\begin{array}{l}\text { Brassica } \\
\text { oleracea L. }\end{array}$ & (LBNR70) & Chou commun & Mkwwar & $\begin{array}{l}\text { Fresh } \\
\text { leaves }\end{array}$ & $\begin{array}{l}\text { Poultice } \\
\text { Juice }\end{array}$ & $\begin{array}{l}\text { Antidiabetic. } \\
\text { Antiulcer. } \\
\text { Against the flu. }\end{array}$ & 82.4 & 27 & 0.032 & \\
\hline & Brassica rapa L. & (LBNR71) & Navet & Left & Roots & $\begin{array}{l}\text { Syrup } \\
\text { Cooked }\end{array}$ & $\begin{array}{l}\text { Heal the flu. } \\
\text { Antidiabetic. (With } \\
\text { the leaves } \\
\text { of Brassica } \\
\text { oleracea). }\end{array}$ & 87.3 & 71 & 0.082 & 0.063 \\
\hline
\end{tabular}




\begin{tabular}{|c|c|c|c|c|c|c|c|c|c|c|c|}
\hline & $\begin{array}{l}\text { Brassica nigra } \\
\text { (L.) W.D.J.Koch }\end{array}$ & (LBNR72) & Moutarde noire & Bu-hammou & $\begin{array}{l}\text { Leafy } \\
\text { stems } \\
\text { seeds }\end{array}$ & $\begin{array}{l}\text { Poultice } \\
\text { Infusion }\end{array}$ & $\begin{array}{l}\text { Antirheumatic. } \\
\text { Stomachic. }\end{array}$ & 91.2 & 64 & 0.077 & \\
\hline & $\begin{array}{l}\text { Capsella bursa } \\
\text { pastoris (L.) } \\
\text { Medik }\end{array}$ & (LBNR73) & Capselle bourse & Tiffaf & Leaves & $\begin{array}{l}\text { Decoction } \\
\text { Infusion }\end{array}$ & $\begin{array}{l}\text { Hemostatic } \\
\text { diuretic }\end{array}$ & 94 & 39 & 0.047 & \\
\hline & $\begin{array}{l}\text { Eruca sativa } \\
\text { Miller }\end{array}$ & (LBNR74) & Roquette & Al girjir & $\begin{array}{l}\text { Fresh } \\
\text { plant } \\
\text { Seeds }\end{array}$ & $\begin{array}{l}\text { Poultice } \\
\text { Lotion } \\
\text { Juice } \\
\text { Powder }\end{array}$ & $\begin{array}{l}\text { Disinfects and heals } \\
\text { wounds. } \\
\text { Promotes hair } \\
\text { growth. } \\
\text { Diuretic. } \\
\text { Antidiabetic. (added } \\
\text { to milk } \\
\text { Fermented and } \\
\text { Lepidium sativum) }\end{array}$ & 78.1 & 102 & 0.123 & \\
\hline & $\begin{array}{l}\text { Lepidium } \\
\text { sativum } \mathrm{L} \text {. }\end{array}$ & (LBNR75) & Cresson alénois & Hab rchad & Seeds & $\begin{array}{l}\text { Decoction } \\
\text { Powder }\end{array}$ & $\begin{array}{l}\text { Treats cough, } \\
\text { asthma, and } \\
\text { bronchitis. } \\
\text { Effective in cases of } \\
\text { infertility. } \\
\text { Antidiabetic }\end{array}$ & 95.6 & 122 & 0.148 & \\
\hline & $\begin{array}{l}\text { Nasturtium } \\
\text { officinale R.Br. }\end{array}$ & (LBNR76) & $\begin{array}{l}\text { Cresson de } \\
\text { fontaine }\end{array}$ & Gernûne & $\begin{array}{l}\text { Leafy } \\
\text { stems }\end{array}$ & Cooked & $\begin{array}{l}\text { Prevent anemia } \\
\text { Heals infections of } \\
\text { the respiratory } \\
\text { system. }\end{array}$ & 83 & 26 & 0.031 & \\
\hline & $\begin{array}{l}\text { Raphanus } \\
\text { sativus L. }\end{array}$ & (LBNR77) & Radis cultivé & Lefjel & $\begin{array}{l}\text { Seeds } \\
\text { Rhizome }\end{array}$ & $\begin{array}{l}\text { Powder } \\
\text { Raw }\end{array}$ & $\begin{array}{l}\text { Antidiabetic. } \\
\text { Stomachic. } \\
\text { Heals liver } \\
\text { problems. }\end{array}$ & 96.4 & 42 & 0.05 & \\
\hline & $\begin{array}{l}\text { Sisymbrium irio } \\
\text { L. }\end{array}$ & (LBNR78) & Roquette jaune & Sibryan & $\begin{array}{l}\text { Leafy } \\
\text { stems } \\
\text { seeds }\end{array}$ & $\begin{array}{l}\text { Infusion } \\
\text { Poultice }\end{array}$ & $\begin{array}{l}\text { Soothes the fever of } \\
\text { typhoid and } \\
\text { smallpox. } \\
\text { Against coughing. } \\
\text { Facilitates the } \\
\text { healing of wounds. }\end{array}$ & 82.3 & 28 & 0.033 & \\
\hline Burseraceae & $\begin{array}{l}\text { Boswellia carterii } \\
\text { Birdy }\end{array}$ & (LBNR79) & $\begin{array}{l}\text { Oliban/ encens } \\
\text { vrai }\end{array}$ & $\begin{array}{l}\text { Salabane/ } \\
\text { loubane }\end{array}$ & Fruits & Decoction & Antidiabetic. & 100 & 82 & 0.09 & 0.09 \\
\hline Buxaceae & $\begin{array}{l}\text { Buxus } \\
\text { sempervirens L. }\end{array}$ & (LBNR80) & Buis commun & Bakss & Leaves & $\begin{array}{l}\text { Infusion } \\
\text { Poultice }\end{array}$ & $\begin{array}{l}\text { Antiseptic } \\
\text { Antirheumatic. }\end{array}$ & 89.7 & 21 & 0.025 & 0.025 \\
\hline Cactaceae & $\begin{array}{l}\text { Opuntia ficus } \\
\text { indica L. (Mill.) }\end{array}$ & (LBNR81) & $\begin{array}{l}\text { Figuier de } \\
\text { barbarie }\end{array}$ & Handiyya & $\begin{array}{l}\text { Fruits } \\
\text { Sap of the } \\
\text { stems }\end{array}$ & $\begin{array}{l}\text { Raw } \\
\text { Cream } \\
\text { Decoction }\end{array}$ & $\begin{array}{l}\text { Soothes } \\
\text { gastrointestinal pain } \\
\text { Softens the hair. }\end{array}$ & 52.8 & 98 & 0.118 & 0.118 \\
\hline
\end{tabular}




\begin{tabular}{|c|c|c|c|c|c|c|c|c|c|c|c|}
\hline & & & & & Flowers & & $\begin{array}{l}\text { Antirheumatic. } \\
\text { Heals bladder and } \\
\text { prostate problems. }\end{array}$ & & & & \\
\hline Capparaceae & $\begin{array}{l}\text { Capparis } \\
\text { spinosa L. }\end{array}$ & (LBNR82) & Câprier & Lkabbar & $\begin{array}{l}\text { Leaves } \\
\text { Roots } \\
\text { Seeds } \\
\text { Fruits }\end{array}$ & $\begin{array}{l}\text { Powder } \\
\text { Decoction } \\
\text { infusion } \\
\text { Powder }\end{array}$ & $\begin{array}{l}\text { Carminative. } \\
\text { Antiulcer. } \\
\text { Diuretic. } \\
\text { Anti-diarrhea } \\
\text { Antidiabetic. } \\
\text { Heals infections of } \\
\text { the respiratory } \\
\text { system. }\end{array}$ & 74.8 & 89 & 0.108 & 0.108 \\
\hline Caprifoliaceae & $\begin{array}{l}\text { Sambucus nigra } \\
\text { L. }\end{array}$ & (LBNR83) & Sureau noir & Sembouqa & $\begin{array}{l}\text { Leaves } \\
\text { Fruits }\end{array}$ & $\begin{array}{l}\text { Decoction } \\
\text { Infusion } \\
\text { Compress }\end{array}$ & $\begin{array}{l}\text { Antidiabetic. } \\
\text { Heals infections of } \\
\text { the respiratory } \\
\text { System. }\end{array}$ & 96.2 & 22 & 0.026 & 0.026 \\
\hline \multirow[t]{3}{*}{ Caryophllaceae } & $\begin{array}{l}\text { Corrigiola } \\
\text { telephiifolia } \\
\text { Pourret. }\end{array}$ & (LBNR84) & $\begin{array}{l}\text { Corrigiole à } \\
\text { Feuilles de } \\
\text { Téléphium }\end{array}$ & Sarghina & Roots & Powder & $\begin{array}{l}\text { Heals infections of } \\
\text { the respiratory } \\
\text { system }\end{array}$ & 100 & 65 & 0.078 & \multirow[t]{3}{*}{0.079} \\
\hline & $\begin{array}{l}\text { Herniaria hirsuta } \\
\text { L. }\end{array}$ & (LBNR85) & Herniaire. & Herrast lehjar & $\begin{array}{l}\text { Whole } \\
\text { plant }\end{array}$ & $\begin{array}{l}\text { Decoction } \\
\text { Infusion }\end{array}$ & $\begin{array}{l}\text { Against kidney } \\
\text { stones. } \\
\text { Reduce bladder } \\
\text { stones. }\end{array}$ & 86.5 & 112 & 0.135 & \\
\hline & $\begin{array}{l}\text { Paronychia } \\
\text { argentea Lam. }\end{array}$ & (LBNR86) & Paronyque & Tahidourt & $\begin{array}{l}\text { Fruits } \\
\text { Leaves }\end{array}$ & Decoction & Heals heart disease. & 97.3 & 22 & 0.026 & \\
\hline \multirow[t]{3}{*}{ Cistaceae } & $\begin{array}{l}\text { Cistus creticus } \\
\text { L. }\end{array}$ & (LBNR87) & Ciste de crête & Irgle & $\begin{array}{l}\text { Leafy } \\
\text { Stems }\end{array}$ & Powder & $\begin{array}{l}\text { Treat irritation of } \\
\text { burns and heal } \\
\text { stomachic. } \\
\text { Against aging and } \\
\text { loosening of the skin }\end{array}$ & 72.2 & 143 & 0.173 & \multirow{3}{*}{0.116} \\
\hline & $\begin{array}{l}\text { Cistus Ladanifer } \\
\text { L. }\end{array}$ & (LBNR88) & Ciste ladanifère & Touzalt & Seeds & $\begin{array}{l}\text { Powder } \\
\text { Infusion }\end{array}$ & $\begin{array}{l}\text { Antidiabetic. } \\
\text { Hemostatic. }\end{array}$ & 98.5 & 79 & 0.095 & \\
\hline & $\begin{array}{l}\text { Cistus } \\
\text { Laurifolius L. }\end{array}$ & (LBNR89) & $\begin{array}{l}\text { Ciste à feuilles } \\
\text { de peuplier }\end{array}$ & Agullid & Seeds & $\begin{array}{l}\text { Powder } \\
\text { Decoction }\end{array}$ & $\begin{array}{l}\text { Antidiabetic. } \\
\text { Decrease sleep } \\
\text { disorders }\end{array}$ & 87.3 & 67 & 0.081 & \\
\hline Convolvulaceae & $\begin{array}{l}\text { Convolvulus } \\
\text { arvensis L. }\end{array}$ & (LBNR90) & $\begin{array}{l}\text { Liseron des } \\
\text { champs }\end{array}$ & Lwayya & $\begin{array}{l}\text { Leaves } \\
\text { Roots }\end{array}$ & Powder & Astringent. & 100 & 85 & 0.103 & 0.103 \\
\hline Cucurbitaceae & $\begin{array}{l}\text { Bryonia dioica } \\
\text { Jacq. }\end{array}$ & (LBNR91) & Bryone dioique & Ineb-eddib & $\begin{array}{l}\text { Stems. } \\
\text { Fruits. }\end{array}$ & Decoction & Antidiabetic. & 100 & 61 & 0.074 & \\
\hline
\end{tabular}




\begin{tabular}{|c|c|c|c|c|c|c|c|c|c|c|c|}
\hline & $\begin{array}{l}\text { Citrullus vulgaris } \\
\text { (L.) Schard. }\end{array}$ & (LBNR92) & Pastèque & Delah & Leaves & $\begin{array}{l}\text { Decoction } \\
\text { maceration }\end{array}$ & Antidiabetic. & 96 & 48 & 0.058 & \multirow[t]{5}{*}{0.083} \\
\hline & Cucumis melo L. & (LBNR93) & Melon & Swihla & Fruits & Raw & $\begin{array}{l}\text { Diuretic. } \\
\text { Promotes the } \\
\text { elimination of kidney } \\
\text { stones. }\end{array}$ & 93.4 & 26 & 0.031 & \\
\hline & $\begin{array}{l}\text { Cucumis sativus } \\
\text { L. }\end{array}$ & (LBNR94) & Concombre & Lkhyar & Seeds & Powder & Antidiabetic. & 100 & 84 & 0.101 & \\
\hline & $\begin{array}{l}\text { Cucurbita } \\
\text { maxima L. }\end{array}$ & (LBNR95) & Courge & Lgraa hamra & $\begin{array}{l}\text { Pulp } \\
\text { Seeds } \\
\text { Fruits }\end{array}$ & $\begin{array}{l}\text { Poultice } \\
\text { Powder } \\
\text { Decoction }\end{array}$ & $\begin{array}{l}\text { Calming burns. } \\
\text { Vermifuge. } \\
\text { Antidiabetic. }\end{array}$ & 93.6 & 109 & 0.132 & \\
\hline & $\begin{array}{l}\text { Lagenaria } \\
\text { siceraria } \\
\text { (Molina) Standl. }\end{array}$ & (LBNR96) & Calebasse & Slawiyya & Fruits & Fumigation & Migraine Therapy & 82 & 86 & 0.104 & \\
\hline \multirow[t]{4}{*}{ Cupressaceae } & $\begin{array}{l}\text { Juniperus } \\
\text { communis L. }\end{array}$ & (LBNR97) & $\begin{array}{l}\text { Genévrier } \\
\text { commun }\end{array}$ & Elarâr - Amzi & Leaves & $\begin{array}{l}\text { Infusion } \\
\text { Powder }\end{array}$ & $\begin{array}{l}\text { Relieves stomach } \\
\text { colic. } \\
\text { Against falling hair. }\end{array}$ & 86.3 & 26 & 0.031 & \multirow{4}{*}{0.032} \\
\hline & $\begin{array}{l}\text { Juniperus } \\
\text { oxycedrus L. }\end{array}$ & (LBNR98) & Genevrier & L arâar chrini & $\begin{array}{l}\text { Stems } \\
\text { Leaves }\end{array}$ & $\begin{array}{l}\text { Decoction } \\
\text { Infusion } \\
\text { Maceration }\end{array}$ & $\begin{array}{l}\text { Stomachic. } \\
\text { Diuretic. } \\
\text { Antidiabetic. }\end{array}$ & 85 & 41 & 0.049 & \\
\hline & $\begin{array}{l}\text { Juniperus } \\
\text { Phoenicea L. }\end{array}$ & (LBNR99) & Genévrier rouge & $\begin{array}{l}\mathrm{L} \text { arâar } \\
\text { El-horr }\end{array}$ & $\begin{array}{l}\text { Aerial } \\
\text { parts }\end{array}$ & $\begin{array}{l}\text { Maceration } \\
\text { Decoction }\end{array}$ & $\begin{array}{l}\text { Antirheumatic. } \\
\text { Aperitif. }\end{array}$ & 94.2 & 19 & 0.023 & \\
\hline & $\begin{array}{l}\text { Tetraclinis } \\
\text { articulata (Vahl) } \\
\text { Masters }\end{array}$ & (LBNR100) & $\begin{array}{l}\text { Thuya de } \\
\text { barbarie }\end{array}$ & $\begin{array}{l}\text { Azougaâ -El } \\
\text { arâar }\end{array}$ & $\begin{array}{l}\text { Leaves } \\
\text { Fruits }\end{array}$ & $\begin{array}{l}\text { Poultice } \\
\text { Compress } \\
\text { Maceration }\end{array}$ & $\begin{array}{l}\text { Migraine Therapy. } \\
\text { Antiseptic. } \\
\text { Antirheumatic. } \\
\text { Calm the pains of } \\
\text { decayed teeth. }\end{array}$ & 78.6 & 22 & 0.026 & \\
\hline Cyperaceae & $\begin{array}{l}\text { Cyperus } \\
\text { Longus L. }\end{array}$ & (LBNR101) & Souchet odorant & $\begin{array}{l}\text { Arouk } \\
\text { Esaaad }\end{array}$ & Roots & Maceration & $\begin{array}{l}\text { Anti-inflammatory } \\
\text { Dermal use } \\
\text { Prevention of } \\
\text { cardiovascular } \\
\text { disorders. }\end{array}$ & 98.4 & 35 & 0.042 & 0.042 \\
\hline Equisetaceae & $\begin{array}{l}\text { Equisetum } \\
\text { ramosissimum } \\
\text { Des }\end{array}$ & (LBNR102) & Prêle & Dayl Ihissan & Stems & Decoction & Antidiabetic. & 100 & 23 & 0.027 & 0.027 \\
\hline Euphorbiaceae & $\begin{array}{l}\text { Euphorbia } \\
\text { echinus Hook.f. } \\
\text { \& Coss. }\end{array}$ & (LBNR103) & $\begin{array}{l}\text { Annuelle } \\
\text { euphorbe }\end{array}$ & Ddaghmus & Stems & $\begin{array}{l}\text { Latex } \\
\text { Powder }\end{array}$ & $\begin{array}{l}\text { Treat eczema. } \\
\text { Antidiabetic (mix } \\
\text { with honey). }\end{array}$ & 92 & 202 & 0.245 & \\
\hline
\end{tabular}




\begin{tabular}{|c|c|c|c|c|c|c|c|c|c|c|c|}
\hline & $\begin{array}{l}\text { Euphorbia } \\
\text { helioscopia L. }\end{array}$ & (LBNR104) & $\begin{array}{l}\text { Euphorbe } \\
\text { réveille-matin }\end{array}$ & $\begin{array}{l}\text { Farbioun } \\
\text { chamss }\end{array}$ & $\begin{array}{l}\text { Leafy } \\
\text { stems }\end{array}$ & Powder & $\begin{array}{l}\text { Heals burns and } \\
\text { some dermal uses at } \\
\text { children }\end{array}$ & 82 & 43 & 0.052 & \multirow[t]{3}{*}{0.169} \\
\hline & $\begin{array}{l}\text { Mercurialis } \\
\text { annua L. }\end{array}$ & (LBNR105) & Annual mercury & $\begin{array}{l}\text { Harryga } \\
\text { Imalsa }\end{array}$ & $\begin{array}{l}\text { Whole } \\
\text { fresh plant }\end{array}$ & $\begin{array}{l}\text { Decoction } \\
\text { Infusion }\end{array}$ & $\begin{array}{l}\text { Antidiabetic. } \\
\text { Traite l'asthme. }\end{array}$ & 96 & 42 & 0.05 & \\
\hline & $\begin{array}{l}\text { Euphorbia } \\
\text { resinifera Berg }\end{array}$ & (LBNR106) & $\begin{array}{l}\text { Euphorbe } \\
\text { résinifère }\end{array}$ & Zeggoum & $\begin{array}{l}\text { Stems } \\
\text { Fruits } \\
\text { Flowers }\end{array}$ & $\begin{array}{l}\text { Poultice } \\
\text { Infusion } \\
\text { Powder }\end{array}$ & $\begin{array}{l}\text { Heals venomous } \\
\text { stings. } \\
\text { Against paralysis } \\
\text { (kneaded with flour } \\
\text { or semolina and egg } \\
\text { white). } \\
\text { abortifacient. } \\
\text { Antidiabetic. }\end{array}$ & 87.4 & 272 & 0.33 & \\
\hline Ericaceae & $\begin{array}{l}\text { Arbutus unedo } \\
\text { L. }\end{array}$ & (LBNR107) & Arbousier & Barnnou & $\begin{array}{l}\text { Bark root } \\
\text { Fruits } \\
\text { Leaves }\end{array}$ & $\begin{array}{l}\text { Infusion } \\
\text { Raw } \\
\text { Powder }\end{array}$ & $\begin{array}{l}\text { Antidiarrheal. } \\
\text { Relieves sore } \\
\text { throats. } \\
\text { Help to sleep. } \\
\text { Antidiabetic. }\end{array}$ & 77 & 123 & 0.149 & 0.149 \\
\hline \multirow[t]{7}{*}{ Fabaceae } & $\begin{array}{l}\text { Acacia } \\
\text { gummifera Willd }\end{array}$ & (LBNR108) & $\begin{array}{l}\text { Gommier du } \\
\text { Maroc }\end{array}$ & Telh & $\begin{array}{l}\text { Leaves } \\
\text { Barks }\end{array}$ & $\begin{array}{l}\text { Poultice } \\
\text { Powder }\end{array}$ & $\begin{array}{l}\text { Heals wounds. } \\
\text { Heals measles. }\end{array}$ & 92.4 & 97 & 0.117 & \multirow{7}{*}{0.123} \\
\hline & $\begin{array}{l}\text { Anagyris foetida } \\
\text { L. }\end{array}$ & (LBNR109) & Anagyre fétide & Foul gnawa & $\begin{array}{l}\text { Seeds } \\
\text { Leaves }\end{array}$ & $\begin{array}{l}\text { Powder } \\
\text { Infusion }\end{array}$ & $\begin{array}{l}\text { Aperitif (mix with } \\
\text { honey). } \\
\text { Antidiabetic. }\end{array}$ & 96.4 & 88 & 0.107 & \\
\hline & Cassia absus L. & (LBNR110) & $\begin{array}{l}\text { Cassia à feuilles } \\
\text { obtuses }\end{array}$ & $\begin{array}{l}\text { El habba } \\
\text { sawdae }\end{array}$ & Seeds & $\begin{array}{l}\text { Powder } \\
\text { Decoction }\end{array}$ & $\begin{array}{l}\text { Antidiabetic. } \\
\text { Diuretics. }\end{array}$ & 82.2 & 69 & 0.083 & \\
\hline & $\begin{array}{l}\text { Ceratonia siliqua } \\
L .\end{array}$ & (LBNR111) & Caroubier & Lkharoub & $\begin{array}{l}\text { Fruits } \\
\text { Leaves }\end{array}$ & $\begin{array}{l}\text { Powder } \\
\text { Infusion }\end{array}$ & $\begin{array}{l}\text { Anti-ulcer. } \\
\text { Emetocathartic. } \\
\text { Laxative. } \\
\text { Antidiabetic. }\end{array}$ & 87.9 & 252 & 0.305 & \\
\hline & $\begin{array}{l}\text { Cicer arietinum } \\
\text { L. }\end{array}$ & (LBNR112) & Pois chiche & Lhomms & Seeds & $\begin{array}{l}\text { Powder } \\
\text { Infusion }\end{array}$ & $\begin{array}{l}\text { Antidiabetic. } \\
\text { Stomachic. } \\
\text { Diuretic and } \\
\text { antispasmodic. }\end{array}$ & 84 & 73 & 0.088 & \\
\hline & $\begin{array}{l}\text { Glycine max (L.) } \\
\text { Merr. }\end{array}$ & (LBNR113) & $\begin{array}{l}\text { Haricot } \\
\text { oléagineux }\end{array}$ & Soja & Seeds & $\begin{array}{l}\text { Decoction } \\
\text { Powder }\end{array}$ & $\begin{array}{l}\text { Antidiabetic. } \\
\text { Increase the weight. }\end{array}$ & 92.3 & 101 & 0.122 & \\
\hline & $\begin{array}{l}\text { Glycyrrhiza } \\
\text { glabra L. }\end{array}$ & (LBNR114) & Réglisse & Aarq ssuss & Roots & $\begin{array}{l}\text { Decoction } \\
\text { Infusion }\end{array}$ & $\begin{array}{l}\text { Against stomach } \\
\text { ulcers } \\
\text { Antidiabetic. } \\
\text { Soothes sore throats }\end{array}$ & 78.8 & 61 & 0.074 & \\
\hline
\end{tabular}




\begin{tabular}{|c|c|c|c|c|c|c|c|c|c|c|c|}
\hline & Lupinus albus $\mathrm{L}$. & (LBNR115) & Lupin blanc & Termis & Seeds & $\begin{array}{l}\text { Powder } \\
\text { Raw }\end{array}$ & $\begin{array}{l}\text { Aperitif } \\
\text { Antidiabetic. }\end{array}$ & 89.3 & 53 & 0.064 & \\
\hline & $\begin{array}{l}\text { Lupinus luteus } \\
\text { L. }\end{array}$ & (LBNR116) & Lupin sauvage & Ssemkala & Seeds & Decoction & $\begin{array}{l}\text { Diuretic } \\
\text { Antidiabetic. } \\
\text { Emmenagogue }\end{array}$ & 94.2 & 71 & 0.086 & \\
\hline & $\begin{array}{l}\text { Medicago sativa } \\
\text { L. }\end{array}$ & (LBNR117) & Luzerne & Fessa & Leaves & $\begin{array}{l}\text { Decoction } \\
\text { Infusion }\end{array}$ & $\begin{array}{l}\text { Antidiabetic. } \\
\text { Reduce cholesterol } \\
\text { levels. } \\
\text { Heals diseases of } \\
\text { the bladder. }\end{array}$ & 73.5 & 152 & 0.184 & \\
\hline & $\begin{array}{l}\text { Ononis spinosa } \\
\text { L. }\end{array}$ & (LBNR118) & Arrète-bœuf & Chanboura & $\begin{array}{l}\text { Whole } \\
\text { plant }\end{array}$ & $\begin{array}{l}\text { Infusion } \\
\text { Decoction } \\
\text { Powder }\end{array}$ & $\begin{array}{l}\text { Diuretic. } \\
\text { Soothes sore throats } \\
\text { Aperitif (mix with } \\
\text { honey). }\end{array}$ & 87.3 & 135 & 0.163 & \\
\hline & $\begin{array}{l}\text { Phaseolus } \\
\text { vulgaris L. }\end{array}$ & (LBNR119) & $\begin{array}{l}\text { Haricot } \\
\text { Vert }\end{array}$ & Loubiyya & Gousse & Raw & Antidiabetic. & 100 & 31 & 0.037 & \\
\hline & $\begin{array}{l}\text { Retama } \\
\text { monosperma } \\
\text { (L.) Boiss }\end{array}$ & (LBNR120) & Rétam blanc & Rtam & $\begin{array}{l}\text { Stems } \\
\text { Roots } \\
\text { Leaves }\end{array}$ & $\begin{array}{l}\text { Infusion } \\
\text { Decoction }\end{array}$ & $\begin{array}{l}\text { Heals diseases of } \\
\text { the gums. } \\
\text { Antidiabetic. } \\
\text { Hypoglycemic. }\end{array}$ & 92.6 & 69 & 0.083 & \\
\hline & $\begin{array}{l}\text { Trifolium } \\
\text { pratense L. }\end{array}$ & (LBNR121) & Trèfle rouge & Rjal Irnb & $\begin{array}{l}\text { Flower } \\
\text { heads }\end{array}$ & $\begin{array}{l}\text { Infusion } \\
\text { Poultice }\end{array}$ & $\begin{array}{l}\text { Diuretic and } \\
\text { antispasmodic. } \\
\text { Calms the itchy skin } \\
\text { Care of dermatitis } \\
\text { and eczema. }\end{array}$ & 78.3 & 84 & 0.101 & \\
\hline & $\begin{array}{l}\text { Trigonella } \\
\text { foenum- } \\
\text { graecum } \mathrm{L} .\end{array}$ & (LBNR122) & Fenugrec & Halba & Seeds & $\begin{array}{l}\text { Maceration } \\
\text { Powder }\end{array}$ & $\begin{array}{l}\text { Aperitif. } \\
\text { Anti-anemic. } \\
\text { Production of breast } \\
\text { milk. } \\
\text { Reduces } \\
\text { gastrointestinal } \\
\text { disorders. } \\
\text { Antidiabetic. }\end{array}$ & 83.6 & 231 & 0.28 & \\
\hline & Vicia faba L. & (LBNR123) & Fève & Lfoul & $\begin{array}{l}\text { Flowers } \\
\text { Seeds }\end{array}$ & Infusion & $\begin{array}{l}\text { Calm the pains of } \\
\text { the kidneys. } \\
\text { Heal the face (mix } \\
\text { with honey). }\end{array}$ & 59 & 71 & 0.086 & \\
\hline Fagaceae & $\begin{array}{l}\text { Quercus } \\
\text { rotundifolia Lam. }\end{array}$ & (LBNR124) & Chêne vert & $\begin{array}{l}\text { Abouhou; } \\
\text { kerrouch }\end{array}$ & Barks & Powder & $\begin{array}{l}\text { Antidiarrheal. } \\
\text { Stomachic (mix with } \\
\text { honey). } \\
\text { Reduce } \\
\text { hemorrhoids. }\end{array}$ & 92.4 & 71 & 0.086 & 0.086 \\
\hline
\end{tabular}




\begin{tabular}{|c|c|c|c|c|c|c|c|c|c|c|c|}
\hline Fumariaceae & $\begin{array}{l}\text { Fumaria } \\
\text { officinalis L. }\end{array}$ & (LBNR125) & Fumeterre & $\begin{array}{l}\text { Lwarda } \\
\text { D'lard }\end{array}$ & $\begin{array}{l}\text { Roots } \\
\text { Fresh } \\
\text { plant }\end{array}$ & $\begin{array}{l}\text { Decoction } \\
\text { Poultice } \\
\text { Infusion }\end{array}$ & $\begin{array}{l}\text { Antidiabetic. } \\
\text { Stomachic. } \\
\text { Laxative. } \\
\text { Heals skin. } \\
\text { Treats eczema. } \\
\text { Fight against nausea } \\
\text { for pregnant women. }\end{array}$ & 56.3 & 43 & 0.052 & 0.052 \\
\hline Gentianaceae & $\begin{array}{l}\text { Centaurium } \\
\text { spicatum (L.) } \\
\text { Fritsch }\end{array}$ & (LBNR126) & Petit centaurée & $\begin{array}{l}\text { Gosset El- } \\
\text { hayya }\end{array}$ & $\begin{array}{l}\text { Whole } \\
\text { plant }\end{array}$ & $\begin{array}{l}\text { Decoction } \\
\text { Infusion } \\
\text { Powder }\end{array}$ & $\begin{array}{l}\text { Antidiabetic. } \\
\text { Anti-hair loss. } \\
\text { Treats } \\
\text { gastrointestinal } \\
\text { disorders. } \\
\text { Aperitif. }\end{array}$ & 67.3 & 38 & 0.046 & 0.046 \\
\hline Geraniaceae & $\begin{array}{l}\text { Pelargonium } \\
\text { roseum l'Hér }\end{array}$ & (LBNR127) & Géranium- rosat & Laattercha & Leaves & Decoction & $\begin{array}{l}\text { Antidiabetic. } \\
\text { Treats burns. }\end{array}$ & 82.5 & 47 & 0.057 & 0.057 \\
\hline Iridaceae & $\begin{array}{l}\text { Crocus sativus } \\
\text { L. }\end{array}$ & (LBNR128) & Safran & Za'farān & Stigmas & $\begin{array}{l}\text { Decoction } \\
\text { Infusion } \\
\text { Massage }\end{array}$ & $\begin{array}{l}\text { Antidiabetic. } \\
\text { Hypotensive. } \\
\text { Stomachic. } \\
\text { Sedative. } \\
\text { Relieves toothaches }\end{array}$ & 94 & 114 & 0.138 & 0.138 \\
\hline Juglandaceae & Juglans regia L. & (LBNR129) & Noyer & Lgargaie & $\begin{array}{l}\text { Barks } \\
\text { Leaves }\end{array}$ & $\begin{array}{l}\text { Chewing } \\
\text { Decoction } \\
\text { Powder }\end{array}$ & $\begin{array}{l}\text { Whiten the teeth. } \\
\text { Antidiabetic. } \\
\text { Heals scars and } \\
\text { wounds. }\end{array}$ & 86.2 & 82 & 0.099 & 0.099 \\
\hline \multirow[t]{4}{*}{ Lamiaceae } & $\begin{array}{l}\text { Ajuga iva (L.) } \\
\text { Schreb. }\end{array}$ & (LBNR130) & Bugle & $\begin{array}{l}\text { Chandgoura; } \\
\text { Timarna }\end{array}$ & $\begin{array}{l}\text { Aerial } \\
\text { parts }\end{array}$ & $\begin{array}{l}\text { Decoction } \\
\text { Maceration } \\
\text { Infusion }\end{array}$ & $\begin{array}{l}\text { Antidiabetic } \\
\text { Heals dental } \\
\text { diseases. } \\
\text { Antidiarrheal. }\end{array}$ & 92.8 & 84 & 0.101 & \\
\hline & $\begin{array}{l}\text { Ballota hirsuta } \\
\text { Benth. }\end{array}$ & (LBNR131) & Ballota poilue & Merrouwt & $\begin{array}{l}\text { Leafy } \\
\text { stems }\end{array}$ & $\begin{array}{l}\text { Decoction } \\
\text { Infusion }\end{array}$ & $\begin{array}{l}\text { Antidiabetic. } \\
\text { Calm cough and } \\
\text { painful spasms of } \\
\text { the digestive tract. }\end{array}$ & 76 & 118 & 0.143 & \\
\hline & $\begin{array}{l}\text { Calamintha } \\
\text { officinalis } \\
\text { Moench }\end{array}$ & (LBNR132) & $\begin{array}{l}\text { Menthe des } \\
\text { montagnes }\end{array}$ & Manta & $\begin{array}{l}\text { Aerial } \\
\text { parts }\end{array}$ & $\begin{array}{l}\text { Decoction } \\
\text { Infusion } \\
\text { Gargles }\end{array}$ & $\begin{array}{l}\text { Antidiabetic. } \\
\text { Calm the intestinal } \\
\text { pains. } \\
\text { Heals the affections } \\
\text { of the mouth. }\end{array}$ & 64.3 & 68 & 0.082 & \\
\hline & $\begin{array}{l}\text { Hyssopus } \\
\text { officinalis L. }\end{array}$ & (LBNR133) & Hysope & Azzoufa & $\begin{array}{l}\text { Flower } \\
\text { heads } \\
\text { Leaves }\end{array}$ & $\begin{array}{l}\text { Infusion } \\
\text { Poultice } \\
\text { Syrup }\end{array}$ & $\begin{array}{l}\text { Stomachic. } \\
\text { Antiseptic. } \\
\text { Sedative. }\end{array}$ & 54.3 & 62 & 0.075 & \\
\hline
\end{tabular}




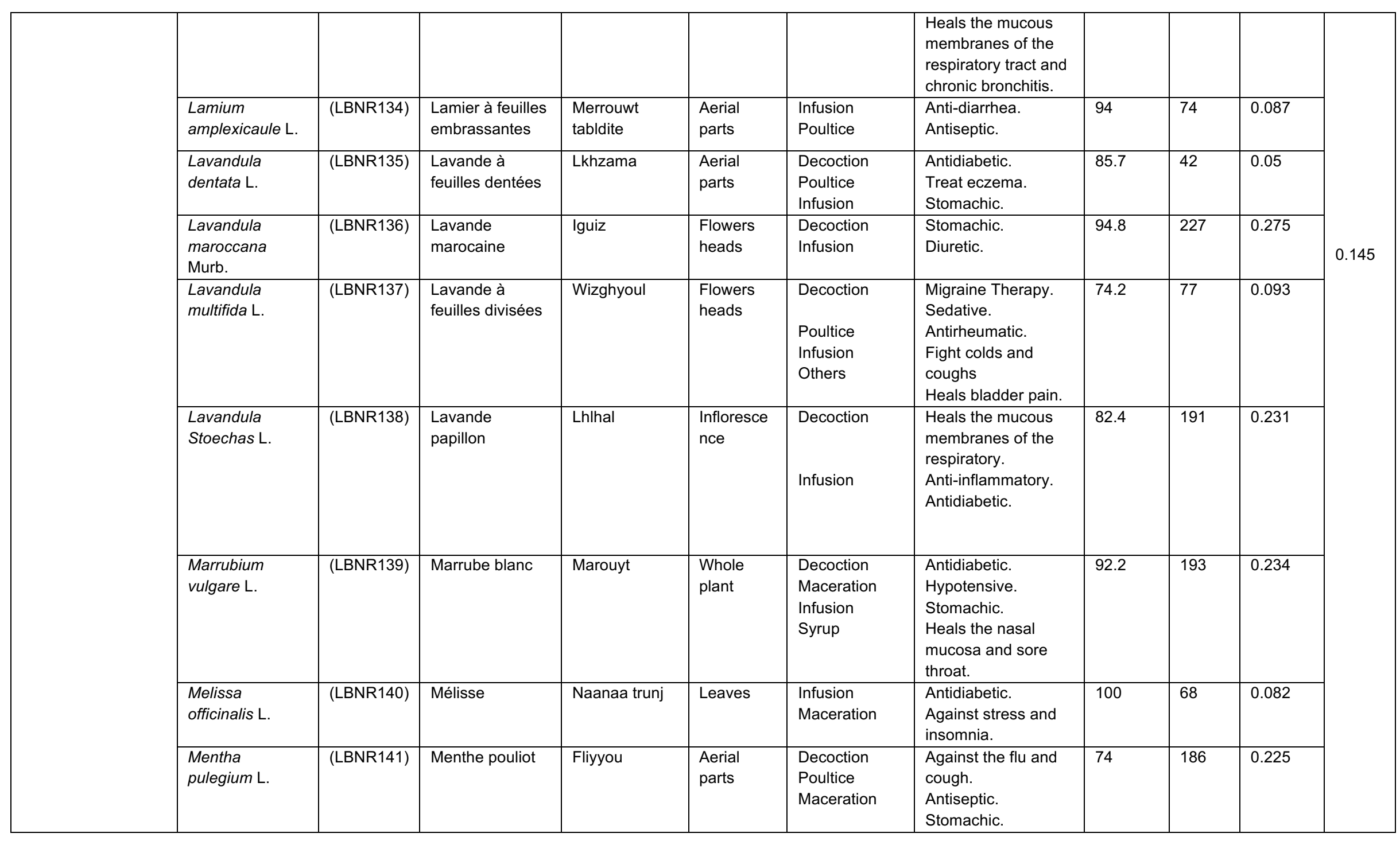









\begin{tabular}{|c|c|c|c|c|c|c|c|c|c|c|c|}
\hline & & & & & & & $\begin{array}{l}\text { Soothes } \\
\text { gastrointestinal } \\
\text { upset. }\end{array}$ & & & & \\
\hline & $\begin{array}{l}\text { Thymus } \\
\text { maroccanus } \\
\text { Ball. }\end{array}$ & (LBNR151) & Thym du Maroc & Azukni & $\begin{array}{l}\text { Leaves } \\
\text { Flowers } \\
\text { heads }\end{array}$ & $\begin{array}{l}\text { Maceration } \\
\text { Infusion }\end{array}$ & $\begin{array}{l}\text { Antidiabetic. } \\
\text { Heals the affections } \\
\text { of the mouth. } \\
\text { Against hair loss (in } \\
\text { olive oil). } \\
\text { Against respiratory } \\
\text { infections. } \\
\text { Stomachic. }\end{array}$ & 89.3 & 128 & 0.155 & \\
\hline & $\begin{array}{l}\text { Thymus } \\
\text { satureioides } \\
\text { Coss. \&Ball. }\end{array}$ & (LBNR152) & $\begin{array}{l}\text { Thym-sarriette } \\
\text { du Maroc }\end{array}$ & Azukni & $\begin{array}{l}\text { Leaves } \\
\text { Flowers } \\
\text { heads }\end{array}$ & $\begin{array}{l}\text { Decoction } \\
\text { Infusion }\end{array}$ & $\begin{array}{l}\text { Antidiabetic. } \\
\text { Heals } \\
\text { bronchopulmonary } \\
\text { affections. } \\
\text { Antiulcer. } \\
\text { Antiseptic. }\end{array}$ & 98 & 214 & 0.259 & \\
\hline & $\begin{array}{l}\text { Thymus vulgaris } \\
\text { L. }\end{array}$ & (LBNR153) & Thym commun & Zaitra & Leaves & $\begin{array}{l}\text { Decoction } \\
\text { /Infusion }\end{array}$ & Antidiabetic. & 100 & 89 & 0.108 & \\
\hline & $\begin{array}{l}\text { Vitex agnus } \\
\text { castus L. }\end{array}$ & (LBNR154) & Arbre à poivre & Anguirf & $\begin{array}{l}\text { Flowers } \\
\text { heads }\end{array}$ & Infusion & $\begin{array}{l}\text { Regularize the } \\
\text { menstrual cycle of } \\
\text { women. }\end{array}$ & 100 & 52 & 0.063 & \\
\hline \multirow[t]{2}{*}{ Lauraceae } & $\begin{array}{l}\text { Cinnamomum } \\
\text { Cassia blum }\end{array}$ & (LBNR155) & Cannelle & El qarfa & Barks & $\begin{array}{l}\text { Decoction } \\
\text { Infusion }\end{array}$ & $\begin{array}{l}\text { Heals liver diseases. } \\
\text { Antidiabetic. } \\
\text { Against menstrual } \\
\text { pains. }\end{array}$ & 89.9 & 89 & 0.108 & \multirow[t]{2}{*}{0.091} \\
\hline & Laurus nobilis L. & (LBNR156) & Laurier noble & Ourak moussa & Leaves & $\begin{array}{l}\text { Decoction } \\
\text { Infusion }\end{array}$ & $\begin{array}{l}\text { Antidiabetic. } \\
\text { Stomachic. }\end{array}$ & 92.5 & 62 & 0.075 & \\
\hline Linaceae & $\begin{array}{l}\text { Linum } \\
\text { usitatissimum L. }\end{array}$ & (LBNR157) & Lin & Zeriaa ketane & Seeds & $\begin{array}{l}\text { Decoction } \\
\text { Powder }\end{array}$ & $\begin{array}{l}\text { Antidiabetic. } \\
\text { For weight gain } \\
\text { (mixed with honey) }\end{array}$ & 86.3 & 92 & 0.111 & 0.111 \\
\hline \multirow[t]{2}{*}{ Lythraceae } & $\begin{array}{l}\text { Lawsonia } \\
\text { inermis } \mathrm{L} \text {. }\end{array}$ & (LBNR158) & Henné & Lḥanna & Leaves & Powder & $\begin{array}{l}\text { Soften skin (mixed } \\
\text { with black soap) } \\
\text { Tint the scalp. }\end{array}$ & 82.4 & 131 & 0.158 & \multirow[t]{2}{*}{0.137} \\
\hline & $\begin{array}{l}\text { Punica } \\
\text { granatum L. }\end{array}$ & (LBNR159) & Grenadier & Rommane & Fruit barks & $\begin{array}{l}\text { Infusion } \\
\text { Powder }\end{array}$ & $\begin{array}{l}\text { Heals bladder } \\
\text { problems and } \\
\text { gastrointestinal pain. } \\
\text { (Mixed with fig and } \\
\text { oleatral flowers). } \\
\text { Antidiabetic. }\end{array}$ & 68 & 96 & 0.116 & \\
\hline
\end{tabular}




\begin{tabular}{|c|c|c|c|c|c|c|c|c|c|c|c|}
\hline & & & & & & & $\begin{array}{l}\text { Softens the hair } \\
\text { (mixed with } \\
\text { Lawsonia inermis). }\end{array}$ & & & & \\
\hline \multirow[t]{4}{*}{ Malvaceae } & $\begin{array}{l}\text { Althaea } \\
\text { Officinalis L. }\end{array}$ & (LBNR160) & Guimauve & Khatmiya & Roots & $\begin{array}{l}\text { Decoction } \\
\text { Maceration } \\
\text { Infusion }\end{array}$ & $\begin{array}{l}\text { Antiulcer. } \\
\text { Heals urinary } \\
\text { disorders. } \\
\text { Against pharyngeal } \\
\text { inflammations. } \\
\text { Antidiarrheal. }\end{array}$ & 87.6 & 68 & 0.082 & \multirow[t]{4}{*}{0.076} \\
\hline & $\begin{array}{l}\text { Hibiscus } \\
\text { Esculentus (L.) } \\
\text { Moench. }\end{array}$ & (LBNR161) & Moench & Lmlokhiyya & $\begin{array}{l}\text { Flowers } \\
\text { Leaves }\end{array}$ & $\begin{array}{l}\text { Decoction } \\
\text { Infusion } \\
\text { Gargle }\end{array}$ & $\begin{array}{l}\text { Antiscorbique } \\
\text { Antidiabetic. } \\
\text { Treats bronchitis. }\end{array}$ & 82.4 & 52 & 0.063 & \\
\hline & $\begin{array}{l}\text { Hibiscus } \\
\text { sabdariffa L. }\end{array}$ & (LBNR162) & Thé rose & Karkadil & $\begin{array}{l}\text { Flowers } \\
\text { Leaves }\end{array}$ & $\begin{array}{l}\text { Infusion } \\
\text { Decoction }\end{array}$ & $\begin{array}{l}\text { Antidiabetic. } \\
\text { Anti-inflammatory. } \\
\text { Diuretic. } \\
\text { Reduce menstrual } \\
\text { pain. }\end{array}$ & 56.2 & 71 & 0.086 & \\
\hline & $\begin{array}{l}\text { Malva parviflora } \\
\text { L. }\end{array}$ & (LBNR163) & $\begin{array}{l}\text { Mauve à petites } \\
\text { fleurs }\end{array}$ & Amzgra & $\begin{array}{l}\text { Aerial } \\
\text { parts }\end{array}$ & $\begin{array}{l}\text { Cooked } \\
\text { Maceration }\end{array}$ & $\begin{array}{l}\text { Laxative. } \\
\text { Antianemic. }\end{array}$ & 96.6 & 63 & 0.076 & \\
\hline Moraceae & Ficus carica L. & (LBNR164) & Figuier & Karmouss & $\begin{array}{l}\text { Leaves } \\
\text { Fruits }\end{array}$ & $\begin{array}{l}\text { Maceration } \\
\text { Evaporation }\end{array}$ & $\begin{array}{l}\text { Antidiabetic. } \\
\text { Treat cough, } \\
\text { bronchitis and } \\
\text { hypotensive (mixed } \\
\text { with evaporated } \\
\text { garlic and olive oil). }\end{array}$ & 89 & 116 & 0.140 & 0.140 \\
\hline \multirow[t]{3}{*}{ Myrtaceae } & $\begin{array}{l}\text { Eucalyptus } \\
\text { globulus Labill. }\end{array}$ & (LBNR165) & Eucalyptus & Eucalyptus & $\begin{array}{l}\text { Barks } \\
\text { Leaves }\end{array}$ & $\begin{array}{l}\text { Decoction } \\
\text { Poultice } \\
\text { Infusion }\end{array}$ & $\begin{array}{l}\text { Antidiabetic. } \\
\text { Treats coughs, flu } \\
\text { and colds. } \\
\text { Antiseptic. }\end{array}$ & 84.2 & 286 & 0.347 & \multirow{3}{*}{0.165} \\
\hline & $\begin{array}{l}\text { Eugenia } \\
\text { caryophyllata } \\
\text { Thunb }\end{array}$ & (LBNR166) & Giroflier & Qronfel & Cloves & $\begin{array}{l}\text { Decoction } \\
\text { Maceration } \\
\text { Infusion }\end{array}$ & $\begin{array}{l}\text { Antidiabetic. } \\
\text { Heals gum disease } \\
\text { (associated with } \\
\text { lavandula officinalis } \\
\text { and juglans regia). } \\
\text { Soothes the pain of } \\
\text { the rules. }\end{array}$ & 69 & 146 & 0.177 & \\
\hline & $\begin{array}{l}\text { Myrtus } \\
\text { communis L. }\end{array}$ & (LBNR167) & Myrte & Rayhane & $\begin{array}{l}\text { Flowers } \\
\text { Leaves }\end{array}$ & $\begin{array}{l}\text { Decoction } \\
\text { Maceration } \\
\text { Infusion }\end{array}$ & $\begin{array}{l}\text { Antidiabetic. } \\
\text { Fight against } \\
\text { dandruff and gives } \\
\text { shine to the hair. }\end{array}$ & 47.3 & 62 & 0.075 & \\
\hline
\end{tabular}




\begin{tabular}{|c|c|c|c|c|c|c|c|c|c|c|c|}
\hline & & & & & & & $\begin{array}{l}\text { Heals cough, and } \\
\text { flu. }\end{array}$ & & & & \\
\hline & $\begin{array}{l}\text { Jasminum } \\
\text { fruticans } \mathrm{L} \text {. }\end{array}$ & (LBNR168) & Jasmin jaune & Yasmin & $\begin{array}{l}\text { Leaves } \\
\text { Flowers }\end{array}$ & $\begin{array}{l}\text { Maceration } \\
\text { Infusion }\end{array}$ & $\begin{array}{l}\text { Antidiabetic. } \\
\text { Relieves intestinal } \\
\text { cramps. } \\
\text { Hypotensive. }\end{array}$ & 56.2 & 52 & 0.063 & \\
\hline Nitrariaceae & $\begin{array}{l}\text { Peganum } \\
\text { harmala L. }\end{array}$ & (LBNR169) & Harmel & L-harmel & Seeds & $\begin{array}{l}\text { Poultice } \\
\text { Fumigation } \\
\text { Infusion }\end{array}$ & $\begin{array}{l}\text { Antirheumatic. } \\
\text { Migraine therapy. } \\
\text { Antidiabetic. }\end{array}$ & 83.9 & 102 & 0.123 & 0.123 \\
\hline \multirow[t]{4}{*}{ Oleaceae } & $\begin{array}{l}\text { Fraxinus } \\
\text { angustifolia } \\
\text { Vahl }\end{array}$ & (LBNR170) & Frêne oxyphylle & Dllam & $\begin{array}{l}\text { Leaves } \\
\text { Bark of the } \\
\text { twigs }\end{array}$ & $\begin{array}{l}\text { Infusion } \\
\text { Maceration }\end{array}$ & $\begin{array}{l}\text { Against urinary } \\
\text { infections. } \\
\text { Antirheumatic. }\end{array}$ & 86.2 & 98 & 0.118 & \multirow{4}{*}{0.096} \\
\hline & $\begin{array}{l}\text { Jasminum } \\
\text { fruticans } \mathrm{L} \text {. }\end{array}$ & (LBNR171) & Jasmin jaune & Yasmin & $\begin{array}{l}\text { Leaves } \\
\text { Flowers }\end{array}$ & $\begin{array}{l}\text { Maceration } \\
\text { Infusion }\end{array}$ & $\begin{array}{l}\text { Antiseptic. } \\
\text { Relieves cramps. } \\
\text { Hypotensive. } \\
\text { Sedative. }\end{array}$ & 94 & 42 & 0.05 & \\
\hline & $\begin{array}{l}\text { Olea europaea } \\
\text { L. }\end{array}$ & (LBNR172) & Olivier & Zaytoun & $\begin{array}{l}\text { Fruits } \\
\text { Leaves }\end{array}$ & $\begin{array}{l}\text { Raw } \\
\text { Decoction } \\
\text { Others }\end{array}$ & $\begin{array}{l}\text { Antidiabetic. } \\
\text { Against oral } \\
\text { inflammation. } \\
\text { Treat otitis. }\end{array}$ & 84.8 & 106 & 0.128 & \\
\hline & $\begin{array}{l}\text { Olea oleaster } \\
\text { Hoffm. \& Link. }\end{array}$ & (LBNR173) & $\begin{array}{l}\text { Oléastre } \\
\text { sauvage }\end{array}$ & Jabouj & Leaves & $\begin{array}{l}\text { Infusion } \\
\text { Decoction }\end{array}$ & $\begin{array}{l}\text { Hypotensive. } \\
\text { Antidiabetic. }\end{array}$ & 48 & 58 & 0.07 & \\
\hline Paeoniaceae & $\begin{array}{l}\text { Paeonia } \\
\text { corallina Retz }\end{array}$ & (LBNR174) & Pivoine coralline & Pantabroun & Flowers & Infusion & $\begin{array}{l}\text { Against coughing } \\
\text { Sedative. }\end{array}$ & 100 & 31 & 0.037 & 0.037 \\
\hline \multirow[t]{2}{*}{ Papaveraceae } & $\begin{array}{l}\text { Fumaria } \\
\text { officinalis L. }\end{array}$ & (LBNR175) & Fumeterre & $\begin{array}{l}\text { Lwarda } \\
\text { D'lard }\end{array}$ & $\begin{array}{l}\text { Roots } \\
\text { Fresh } \\
\text { plant }\end{array}$ & $\begin{array}{l}\text { Decoction } \\
\text { Poultice } \\
\text { Infusion }\end{array}$ & $\begin{array}{l}\text { Antidiabetic. } \\
\text { Stomachic. } \\
\text { Laxative. } \\
\text { Heals eczema. } \\
\text { Fight against nausea } \\
\text { for pregnant women. }\end{array}$ & 57.8 & 68 & 0.082 & \multirow[t]{2}{*}{0.096} \\
\hline & $\begin{array}{l}\text { Papaver rhoeas } \\
\text { L. }\end{array}$ & (LBNR176) & Pavot hybride & Belnaaman & $\begin{array}{l}\text { Seeds } \\
\text { Flowers }\end{array}$ & $\begin{array}{l}\text { Decoction } \\
\text { Powder } \\
\text { Infusion }\end{array}$ & $\begin{array}{l}\text { Against dry cough. } \\
\text { Calm stomach ache. } \\
\text { Eliminate fatigue. } \\
\text { Calm sore throat } \\
\text { and cough in } \\
\text { children. }\end{array}$ & 93.5 & 92 & 0.111 & \\
\hline Pedaliaceae & $\begin{array}{l}\text { Sesamum } \\
\text { indicum } \mathrm{L} \text {. }\end{array}$ & (LBNR177) & Sésame & Zenjlan & Seeds & $\begin{array}{l}\text { Decoction } \\
\text { Infusion } \\
\text { Powder }\end{array}$ & $\begin{array}{l}\text { Antidiabetic. } \\
\text { Diuretics. }\end{array}$ & 75.5 & 78 & 0.094 & 0.094 \\
\hline
\end{tabular}




\begin{tabular}{|c|c|c|c|c|c|c|c|c|c|c|c|}
\hline & & & & & & & $\begin{array}{l}\text { Against } \\
\text { gastrointestinal } \\
\text { disorders. }\end{array}$ & & & & \\
\hline \multirow[t]{2}{*}{ Pinaceae } & $\begin{array}{l}\text { Cedrus } \\
\text { Atlantica } \\
\text { Manetti ex } \\
\text { (Endl.) }\end{array}$ & (LBNR178) & Cèdre de l'Atlas & Arz & Barks & $\begin{array}{l}\text { Decoction } \\
\text { Infusion } \\
\text { Maceration } \\
\text { Powder }\end{array}$ & $\begin{array}{l}\text { Fights flu, coughs } \\
\text { and bronchitis. } \\
\text { Heals urinary tract } \\
\text { infections. } \\
\text { Sedative. } \\
\text { Against hair loss (in } \\
\text { olive oil). }\end{array}$ & 51 & 31 & 0.037 & \multirow[t]{2}{*}{0.053} \\
\hline & $\begin{array}{l}\text { Pinus } \\
\text { halepensis Mill. }\end{array}$ & (LBNR179) & Pin d'Alep & $\begin{array}{l}\text { Snaoibar- } \\
\text { Tayda }\end{array}$ & $\begin{array}{l}\text { Leaves } \\
\text { Barks }\end{array}$ & $\begin{array}{l}\text { Infusion } \\
\text { Powder }\end{array}$ & $\begin{array}{l}\text { Soothes catarrhal } \\
\text { affections of the } \\
\text { respiratory system. } \\
\text { Heals burns of the } \\
\text { skin (with clarified } \\
\text { butter). }\end{array}$ & 72.2 & 58 & 0.07 & \\
\hline \multirow[t]{3}{*}{ Plantaginaceae } & $\begin{array}{l}\text { Globularia } \\
\text { alypum L. }\end{array}$ & (LBNR180) & $\begin{array}{l}\text { Globulaire } \\
\text { buissonnante }\end{array}$ & Taslgha & $\begin{array}{l}\text { Flower } \\
\text { heads }\end{array}$ & $\begin{array}{l}\text { Decoction } \\
\text { Infusion }\end{array}$ & $\begin{array}{l}\text { Antidiabetic. } \\
\text { Antiulcer }\end{array}$ & 86.5 & 77 & 0.093 & \multirow{3}{*}{0.108} \\
\hline & $\begin{array}{l}\text { Plantago major } \\
\text { L. }\end{array}$ & (LBNR181) & Grand plantain & Lmassas & $\begin{array}{l}\text { Whole } \\
\text { plant }\end{array}$ & $\begin{array}{l}\text { Poultice } \\
\text { Infusion } \\
\text { Powder }\end{array}$ & $\begin{array}{l}\text { Heal eczema. } \\
\text { Against asthma and } \\
\text { eliminates bronchial } \\
\text { mucous } \\
\text { membranes. } \\
\text { Promotes the } \\
\text { healing of wounds. }\end{array}$ & 74.2 & 71 & 0.086 & \\
\hline & $\begin{array}{l}\text { Plantago ovata } \\
\text { Forsskal }\end{array}$ & (LBNR182) & Ispaghul & $\begin{array}{l}\text { Zriaat } \\
\text { zrktouna }\end{array}$ & Seeds & $\begin{array}{l}\text { Decoction } \\
\text { Powder }\end{array}$ & $\begin{array}{l}\text { Diuretic. } \\
\text { Hypotensive. }\end{array}$ & 98.2 & 121 & 0.146 & \\
\hline \multirow[t]{5}{*}{ Poaceae } & Arundo donax L. & (LBNR183) & $\begin{array}{l}\text { Canne de } \\
\text { provence }\end{array}$ & Ksab & Roots & Powder & $\begin{array}{l}\text { Against hair loss (in } \\
\text { olive oil). }\end{array}$ & 100 & 156 & 0.183 & \\
\hline & Avena sativa L. & (LBNR184) & Avoine cultivée & Achoufane & Seeds & Infusion & Antidiabetic. & 100 & 52 & 0.063 & \\
\hline & Avena sterilis L. & (LBNR185) & Avoine & Askoune & Fruits & $\begin{array}{l}\text { Decoction } \\
\text { Powder }\end{array}$ & $\begin{array}{l}\text { Stomachic. } \\
\text { Antidiabetic (mixed } \\
\text { with the flowers of } \\
\text { zea mays). }\end{array}$ & 88.8 & 99 & 0.12 & \\
\hline & $\begin{array}{l}\text { Cynodon } \\
\text { dactylon (L.) } \\
\text { Pers. }\end{array}$ & (LBNR186) & Cynodon & Njem & Roots & $\begin{array}{l}\text { Decoction } \\
\text { Powder }\end{array}$ & $\begin{array}{l}\text { Stops nosebleeds. } \\
\text { Calms burns and } \\
\text { heals wounds. }\end{array}$ & 92.3 & 84 & 0.101 & \\
\hline & $\begin{array}{l}\text { Hordeum } \\
\text { vulgare L. }\end{array}$ & (LBNR187) & Orge & Chaâir & Seeds & Maceration & $\begin{array}{l}\text { Antidiabetic (mixed } \\
\text { with fenugreek } \\
\text { powder). }\end{array}$ & 100 & 62 & 0.075 & 0.116 \\
\hline
\end{tabular}




\begin{tabular}{|c|c|c|c|c|c|c|c|c|c|c|c|}
\hline & $\begin{array}{l}\text { Lolium perenne } \\
\text { L. }\end{array}$ & (LBNR188) & Ivraie vivace & Zouane & Seeds & $\begin{array}{l}\text { Decoction } \\
\text { infusion }\end{array}$ & Antidiabetic. & 72.4 & 69 & 0.083 & \\
\hline & $\begin{array}{l}\text { Panicum } \\
\text { miliaceum L. }\end{array}$ & (LBNR189) & Millet commun & Tafsout & Seeds & $\begin{array}{l}\text { Decoction } \\
\text { Infusion }\end{array}$ & $\begin{array}{l}\text { Antidiabetic. } \\
\text { Promotes digestion. }\end{array}$ & 92.6 & 81 & 0.098 & \\
\hline & $\begin{array}{l}\text { Pennisetum } \\
\text { glaucum (L.) R. } \\
\mathrm{Br}\end{array}$ & (LBNR190) & Millet & İllân & Seeds & Powder & $\begin{array}{l}\text { Antianemic. } \\
\text { Antidiabetic. }\end{array}$ & 94 & 203 & 0.246 & \\
\hline & $\begin{array}{l}\text { Phalaris } \\
\text { canariensis L. }\end{array}$ & (LBNR191) & $\begin{array}{l}\text { Alpiste des } \\
\text { canaries }\end{array}$ & Zwan abiyad & Seeds & Powder & Antidiabetic. & 100 & 69 & 0.083 & \\
\hline & $\begin{array}{l}\text { Phragmites } \\
\text { australis (Cav) } \\
\text { Steud. }\end{array}$ & (LBNR192) & Roseau & L-qasseb & $\begin{array}{l}\text { Leaves } \\
\text { Roots }\end{array}$ & $\begin{array}{l}\text { Decoction } \\
\text { Powder }\end{array}$ & $\begin{array}{l}\text { Against bronchitis. } \\
\text { Anti-hair loss. }\end{array}$ & 72.3 & 113 & 0.137 & \\
\hline & $\begin{array}{l}\text { Triticum } \\
\text { Durum Desf. }\end{array}$ & (LBNR193) & Blé & Lkamh & Seeds & Maceration & $\begin{array}{l}\text { Antidiabetic (mix } \\
\text { with Coriandrum } \\
\text { sativum and } \\
\text { Trigonella foenum- } \\
\text { graecum). } \\
\text { Anti-hair loss. }\end{array}$ & 78.8 & 81 & 0.098 & \\
\hline & Zea mays L. & (LBNR194) & Mais & Draa & $\begin{array}{l}\text { Stigma of } \\
\text { corn }\end{array}$ & $\begin{array}{l}\text { Infusion } \\
\text { Decoction } \\
\text { Powder }\end{array}$ & $\begin{array}{l}\text { Hair care. } \\
\text { Against disorders of } \\
\text { the bladder } \\
\text { Antianemic. }\end{array}$ & 94 & 102 & 0.123 & \\
\hline Polygalaceae & $\begin{array}{l}\text { Polygala } \\
\text { rupestris Pourret }\end{array}$ & (LBNR195) & $\begin{array}{l}\text { Polygala des } \\
\text { rochers }\end{array}$ & Laachba dlhlib & Roots & Decoction & $\begin{array}{l}\text { Heals asthma } \\
\text { attacks. } \\
\text { Laxative. }\end{array}$ & 100 & 101 & 0.122 & 0.122 \\
\hline \multirow[t]{2}{*}{ Polygonaceae } & $\begin{array}{l}\text { Rumex } \\
\text { bucephalophoru } \\
\text { s L. }\end{array}$ & (LBNR196) & $\begin{array}{l}\text { Rumex tête de } \\
\text { boeuf }\end{array}$ & Tismmam & $\begin{array}{l}\text { Whole } \\
\text { plant }\end{array}$ & $\begin{array}{l}\text { Decoction } \\
\text { Infusion } \\
\text { Compress }\end{array}$ & $\begin{array}{l}\text { Aperitif. } \\
\text { Soothes the pain of } \\
\text { the bladder. } \\
\text { Antiseptic. }\end{array}$ & 89.6 & 89 & 0.108 & \multirow[t]{2}{*}{0.1} \\
\hline & $\begin{array}{l}\text { Rumex pulcher } \\
\text { L. }\end{array}$ & (LBNR197) & Oseil sauvage & Hommayda & $\begin{array}{l}\text { Whole } \\
\text { plant }\end{array}$ & $\begin{array}{l}\text { Decoction } \\
\text { Infusion }\end{array}$ & $\begin{array}{l}\text { Against bladder } \\
\text { pain. } \\
\text { Stomachic. }\end{array}$ & 83.3 & 76 & 0.092 & \\
\hline Portulacaceae & $\begin{array}{l}\text { Portulaca } \\
\text { oleraceae L. }\end{array}$ & (LBNR198) & Pourpier potager & Rejla & $\begin{array}{l}\text { Aerial } \\
\text { parts }\end{array}$ & $\begin{array}{l}\text { Infusion } \\
\text { Decoction } \\
\text { Cooked }\end{array}$ & $\begin{array}{l}\text { Laxative. } \\
\text { Soothes the pain of } \\
\text { the bladder. } \\
\text { Antidiabetic. }\end{array}$ & 72.2 & 102 & 0.123 & 0.123 \\
\hline Primulaceae & $\begin{array}{l}\text { Anagallis } \\
\text { arvensis } \mathrm{L} .\end{array}$ & (LBNR199) & $\begin{array}{l}\text { Mouron des } \\
\text { champs }\end{array}$ & $\begin{array}{l}\text { Chahmet } \\
\text { l'falüss }\end{array}$ & $\begin{array}{l}\text { Leaves } \\
\text { Stems }\end{array}$ & $\begin{array}{l}\text { Decoction } \\
\text { Poultice }\end{array}$ & $\begin{array}{l}\text { Against kidney and } \\
\text { bladder stones. } \\
\text { Heals skin diseases. }\end{array}$ & 100 & 62 & 0.075 & 0.075 \\
\hline
\end{tabular}




\begin{tabular}{|c|c|c|c|c|c|c|c|c|c|c|c|}
\hline \multirow[t]{4}{*}{ Ranunculaceae } & $\begin{array}{l}\text { Clematis } \\
\text { flammula } \mathrm{L} .\end{array}$ & (LBNR201) & $\begin{array}{l}\text { Clématite } \\
\text { brûlante }\end{array}$ & Nar el-barda & $\begin{array}{l}\text { Aerial } \\
\text { parts }\end{array}$ & Poultice & Antirheumatic. & 83.3 & 91 & 0.11 & \multirow{4}{*}{0.133} \\
\hline & $\begin{array}{l}\text { Delphinium } \\
\text { pentagynum } \\
\text { Lam }\end{array}$ & (LBNR202) & $\begin{array}{l}\text { Dauphinelle } \\
\text { staphysaigre }\end{array}$ & Habbet râs & Leaves & $\begin{array}{l}\text { Decoction } \\
\text { Poultice }\end{array}$ & $\begin{array}{l}\text { Antidiarrheal. } \\
\text { Anti-lice (mixed with } \\
\text { olive oil). }\end{array}$ & 96.2 & 71 & 0.086 & \\
\hline & Nigella sativa L. & (LBNR203) & Nigelle & Sanouje & Seeds & Powder & $\begin{array}{l}\text { Antidiabetic. } \\
\text { Stomachic. } \\
\text { Treats liver diseases } \\
\text { (mixed with honey). }\end{array}$ & 78.5 & 87 & 0.105 & \\
\hline & $\begin{array}{l}\text { Ranunculus } \\
\text { bullatus } \mathrm{L} .\end{array}$ & (LBNR204) & $\begin{array}{l}\text { Renoncule } \\
\text { boursouflée }\end{array}$ & $\begin{array}{l}\text { Mrniss, Wdan } \\
\text { Alhalouf }\end{array}$ & $\begin{array}{l}\text { Roots } \\
\text { Leaves }\end{array}$ & $\begin{array}{l}\text { Decoction } \\
\text { Poultice }\end{array}$ & $\begin{array}{l}\text { Regulates the rules } \\
\text { and hormones in } \\
\text { women. } \\
\text { Stomachic. } \\
\text { Facilitates childbirth. }\end{array}$ & 64 & 131 & 0.158 & \\
\hline Resedaceae & Reseda alba $L$. & (LBNR205) & Réséda blanc & Tabaddit & Leaves & Infusion & $\begin{array}{l}\text { Antidiarrheal. } \\
\text { Diuretic. }\end{array}$ & 100 & 101 & 0.122 & 0.122 \\
\hline Rhamnaceae & $\begin{array}{l}\text { Ziziphus lotus } \\
\text { (L.) Lam. }\end{array}$ & (LBNR206) & Jujubier & $\begin{array}{l}\text { Ssedra, } \\
\text { Azgour }\end{array}$ & $\begin{array}{l}\text { Leaves } \\
\text { Fruits }\end{array}$ & $\begin{array}{l}\text { Decoction } \\
\text { Powder } \\
\text { Infusion }\end{array}$ & $\begin{array}{l}\text { Antidiarrheal. } \\
\text { Antiulcer. } \\
\text { Aperitif. } \\
\text { Antidiabetic. } \\
\text { Promotes the } \\
\text { healing of wounds. }\end{array}$ & 87 & 131 & 0.158 & 0.158 \\
\hline \multirow[t]{6}{*}{ Rosaceae } & $\begin{array}{l}\text { Crataegus } \\
\text { monogyna } \\
\text { Jacquin }\end{array}$ & (LBNR207) & $\begin{array}{l}\text { Crataegus à } \\
\text { épines aiguës. }\end{array}$ & Azzairour & $\begin{array}{l}\text { Flower } \\
\text { heads. }\end{array}$ & $\begin{array}{l}\text { Powder } \\
\text { Infusion }\end{array}$ & $\begin{array}{l}\text { Against heart and } \\
\text { respiratory problems } \\
\text { (mixed with honey) } \\
\text { Promotes the } \\
\text { circulation of blood. }\end{array}$ & 78.6 & 99 & 0.12 & \multirow{6}{*}{0.126} \\
\hline & $\begin{array}{l}\text { Cydonia oblonga } \\
\text { Mill. }\end{array}$ & (LBNR208) & Cognassier & Sferjel & $\begin{array}{l}\text { Leaves } \\
\text { seeds }\end{array}$ & $\begin{array}{l}\text { Friction } \\
\text { Gargle }\end{array}$ & $\begin{array}{l}\text { Heals hemorrhoids. } \\
\text { Heals sore throats. }\end{array}$ & 92.8 & 52 & 0.063 & \\
\hline & $\begin{array}{l}\text { Prunus } \\
\text { amygdalus } \\
\text { Batsh. } \\
\end{array}$ & (LBNR209) & Amandier amer & Loz Iharr & Leaves & $\begin{array}{l}\text { Decoction } \\
\text { Infusion }\end{array}$ & $\begin{array}{l}\text { Antidiabetic. } \\
\text { Laxative } \\
\text { Soothes cough }\end{array}$ & 72.9 & 82 & 0.099 & \\
\hline & $\begin{array}{l}\text { Prunus } \\
\text { armeniaca L. }\end{array}$ & (LBNR210) & Abricotier & Imchmach & Fruits & Powders & $\begin{array}{l}\text { Antidiabetic. } \\
\text { Antidiarrheal. }\end{array}$ & 94 & 71 & 0.086 & \\
\hline & $\begin{array}{l}\text { Prunus } \\
\text { domestica L. }\end{array}$ & (LBNR211) & Prunier & Lbrkouk & $\begin{array}{l}\text { Leaves } \\
\text { Fruits }\end{array}$ & Raw & $\begin{array}{l}\text { Laxative. } \\
\text { Diuretic. }\end{array}$ & 89.2 & 26 & 0.031 & \\
\hline & $\begin{array}{l}\text { Prunus dulcis } \\
\text { Mill.) D.A.Webb }\end{array}$ & (LBNR212) & Amandier & Louz & $\begin{array}{l}\text { Leaves } \\
\text { Fruits }\end{array}$ & $\begin{array}{l}\text { Infusion } \\
\text { Powder }\end{array}$ & $\begin{array}{l}\text { Calm the cough. } \\
\text { Laxative. }\end{array}$ & 91.9 & 68 & 0.082 & \\
\hline
\end{tabular}




\begin{tabular}{|c|c|c|c|c|c|c|c|c|c|c|c|}
\hline & Rosa canina $L$. & (LBNR213) & Églantier & Nisrîn & $\begin{array}{l}\text { Leaves. } \\
\text { Fruits. } \\
\text { Flower } \\
\text { buds. }\end{array}$ & $\begin{array}{l}\text { Infusion } \\
\text { Powder }\end{array}$ & $\begin{array}{l}\text { Antidiarrheal. } \\
\text { Antianemic. } \\
\text { Heals burns (mixed } \\
\text { with olive oil). }\end{array}$ & 89.3 & 76 & 0.092 & \\
\hline & $\begin{array}{l}\text { Rosa centifolia } \\
\text { Mill }\end{array}$ & (LBNR214) & Rosecentfeuilles & El ward & Dried buds & $\begin{array}{l}\text { Decoction } \\
\text { Maceration }\end{array}$ & $\begin{array}{l}\text { Stomachic. } \\
\text { Treat the sores. }\end{array}$ & 97.3 & 132 & 0.16 & \\
\hline & $\begin{array}{l}\text { Rosa } \\
\text { damascena Mill. }\end{array}$ & (LBNR215) & $\begin{array}{l}\text { Rose rouge de } \\
\text { Damas }\end{array}$ & $\begin{array}{l}\text { El ward el } \\
\text { beldi }\end{array}$ & Flowers & $\begin{array}{l}\text { Compress } \\
\text { Powder }\end{array}$ & $\begin{array}{l}\text { Antiseptic. } \\
\text { Soften the hair. }\end{array}$ & 94 & 184 & 0.223 & \\
\hline & Rosa gallica L. & (LBNR216) & $\begin{array}{l}\text { Rosa rubra } \\
\text { Black }\end{array}$ & El ward & $\begin{array}{l}\text { Flower } \\
\text { buds. }\end{array}$ & Infusion & $\begin{array}{l}\text { Treats scars. } \\
\text { Hemostatic. }\end{array}$ & 87.2 & 163 & 0.197 & \\
\hline & $\begin{array}{l}\text { Rubus ulmifolius } \\
\text { Schott. }\end{array}$ & (LBNR217) & Ronce & $\begin{array}{l}\text { Laallik, } \\
\text { Tabgha }\end{array}$ & $\begin{array}{l}\text { Fruits } \\
\text { Flowers } \\
\text { Leaves }\end{array}$ & $\begin{array}{l}\text { Infusion } \\
\text { Decoction } \\
\text { Infusion }\end{array}$ & $\begin{array}{l}\text { Antidiabetic. } \\
\text { Anti-wrinkle. } \\
\text { Against the pain of } \\
\text { menstruation. }\end{array}$ & 56 & 225 & 0.273 & \\
\hline & $\begin{array}{l}\text { Sanguisorba } \\
\text { minor Scop. }\end{array}$ & $\begin{array}{l}\text { (LBNR218) } \\
\text { (LBNR }\end{array}$ & Sanguisorbe & $\begin{array}{l}\text { Faggass } \\
\text { laklab }\end{array}$ & $\begin{array}{l}\text { Aerial } \\
\text { parts } \\
\text { Roots }\end{array}$ & $\begin{array}{l}\text { Infusion } \\
\text { Gargle } \\
\text { Powder }\end{array}$ & $\begin{array}{l}\text { Antidiarrheal. } \\
\text { Heals } \\
\text { gastrointestinal } \\
\text { disorders. } \\
\text { Fights gum } \\
\text { infections and } \\
\text { infectious tonsillitis. } \\
\text { Relieves burn and } \\
\text { heals eczema. }\end{array}$ & 66.4 & 78 & 0.094 & \\
\hline Rubiaceae & $\begin{array}{l}\text { Rubia tinctorum } \\
\text { L. }\end{array}$ & (LBNR219) & Garance & $\begin{array}{l}\text { Fouwwal } \\
\text { Taroubiya }\end{array}$ & $\begin{array}{l}\text { Roots } \\
\text { Leaves }\end{array}$ & $\begin{array}{l}\text { Decoction } \\
\text { Cooked }\end{array}$ & $\begin{array}{l}\text { Against jaundice and } \\
\text { liver diseases. } \\
\text { Antianemic. }\end{array}$ & 85.9 & 126 & 0.152 & 0.152 \\
\hline \multirow[t]{4}{*}{ Rutaceae } & $\begin{array}{l}\text { Citrus aurantium } \\
\text { L. }\end{array}$ & (LBNR220) & Oranger amer & Larange & $\begin{array}{l}\text { Fruits } \\
\text { Flowers }\end{array}$ & $\begin{array}{l}\text { Juice } \\
\text { Poultice }\end{array}$ & $\begin{array}{l}\text { Antidiabetic. } \\
\text { Promotes blood } \\
\text { circulation. } \\
\text { Heal the sunburns. }\end{array}$ & 79.4 & 108 & 0.137 & \multirow{4}{*}{0.099} \\
\hline & $\begin{array}{l}\text { Citrus limon (L.) } \\
\text { Burm.f. }\end{array}$ & (LBNR221) & Citronier & Leymun & $\begin{array}{l}\text { Fruits } \\
\text { Barks }\end{array}$ & $\begin{array}{l}\text { Gargle } \\
\text { Juice }\end{array}$ & $\begin{array}{l}\text { Against angina. } \\
\text { Heal the intestinal } \\
\text { pain. }\end{array}$ & 100 & 76 & 0.092 & \\
\hline & Citrus limette & (LBNR22) & Bergamia & El hamed Ibldi & Flowers & Infusion & $\begin{array}{l}\text { Antidiabetic. } \\
\text { Anti-migraine. }\end{array}$ & 94 & 58 & 0.07 & \\
\hline & $\begin{array}{l}\text { Citrus sinensis } \\
\text { (L.) Osbeck }\end{array}$ & (LBNR223) & Oranger doux & Leymun & $\begin{array}{l}\text { Fruits } \\
\text { barks } \\
\text { Fruits }\end{array}$ & $\begin{array}{l}\text { Powder } \\
\text { Cooked }\end{array}$ & $\begin{array}{l}\text { Treat acne (mixed } \\
\text { with a } \\
\text { Unsweetened } \\
\text { natural yoghurt). } \\
\text { Against the flu. }\end{array}$ & 82.8 & 68 & 0.082 & \\
\hline
\end{tabular}




\begin{tabular}{|c|c|c|c|c|c|c|c|c|c|c|c|}
\hline & $\begin{array}{l}\text { Ruta graveolens } \\
\text { L. }\end{array}$ & (LBNR224) & Rue officinale & L-Fijel & Roots & Decoction & $\begin{array}{l}\text { Antidiabetic. } \\
\text { Relieves sciatic. } \\
\text { Promote digestion }\end{array}$ & 91.5 & 96 & 0.116 & \\
\hline & $\begin{array}{l}\text { Ruta Montana } \\
\text { (L.) L. }\end{array}$ & (LBNR225) & Rue sauvage & Awrma & $\begin{array}{l}\text { Roots } \\
\text { Flower }\end{array}$ & $\begin{array}{l}\text { Decoction } \\
\text { Maceration } \\
\text { Fumigation }\end{array}$ & $\begin{array}{l}\text { Stomachic. } \\
\text { Heals liver diseases. } \\
\text { Antidiabetic. } \\
\text { Fight against lice (in } \\
\text { vinegar). } \\
\text { For the insomnia of } \\
\text { children. }\end{array}$ & 51.7 & 102 & 0.123 & \\
\hline Schisandraceae & $\begin{array}{l}\text { Illicium verum } \\
\text { Hook. F }\end{array}$ & (LBNR226) & Anis étoilé & Badiana & $\begin{array}{l}\text { Fruits } \\
\text { Leaves }\end{array}$ & $\begin{array}{l}\text { Decoction } \\
\text { Powder }\end{array}$ & $\begin{array}{l}\text { Against digestive } \\
\text { problems and in } \\
\text { particular bloating. } \\
\text { Migraine Therapy. }\end{array}$ & 74.2 & 68 & 0.082 & 0.082 \\
\hline \multirow[t]{8}{*}{ Solanaceae } & $\begin{array}{l}\text { Capsicum } \\
\text { annuum } \mathrm{L} .\end{array}$ & (LBNR227) & Poivron & Felfla & Fruits & $\begin{array}{l}\text { Cooked } \\
\text { Other }\end{array}$ & $\begin{array}{l}\text { Aperitif. } \\
\text { Antiseptic. } \\
\text { Diuretic. } \\
\text { Antidiabetic }\end{array}$ & 86.3 & 61 & 0.074 & \multirow{8}{*}{0.094} \\
\hline & $\begin{array}{l}\text { Capsicum } \\
\text { frutescens } \mathrm{L} .\end{array}$ & (LBNR228) & $\begin{array}{l}\text { Poivre de } \\
\text { cayenne }\end{array}$ & FIfla harra & Fruits & $\begin{array}{l}\text { Decoction } \\
\text { Powder }\end{array}$ & $\begin{array}{l}\text { Vermifuge (in olive } \\
\text { oil). } \\
\text { Strengthens the hair } \\
\text { (in apple vinegar). }\end{array}$ & 82 & 87 & 0.105 & \\
\hline & $\begin{array}{l}\text { Datura } \\
\text { stramonium L. }\end{array}$ & (LBNR229) & Stramoine & Lkoukhra & Seeds & $\begin{array}{l}\text { Decoction } \\
\text { Powder }\end{array}$ & $\begin{array}{l}\text { Soothes the cough. } \\
\text { Antiseptic. } \\
\text { For weight gain (with } \\
\text { couscous beans). }\end{array}$ & 92 & 79 & 0.095 & \\
\hline & $\begin{array}{l}\text { Hyoscyamus } \\
\text { niger L. }\end{array}$ & (LBNR230) & Jusquiame & Guingate & $\begin{array}{l}\text { Leaves } \\
\text { Flowers }\end{array}$ & Decoction & $\begin{array}{l}\text { Relieves sciatic or } \\
\text { rheumatic pains. } \\
\text { Treat eczema. }\end{array}$ & 94 & 146 & 0.177 & \\
\hline & $\begin{array}{l}\text { Solanum } \\
\text { dulcamara L. }\end{array}$ & (LBNR231) & Morelle & Ainab dib & $\begin{array}{l}\text { Stems } \\
\text { Leaves }\end{array}$ & $\begin{array}{l}\text { Poultice } \\
\text { Decoction } \\
\text { Powder }\end{array}$ & $\begin{array}{l}\text { Anti-rheumatic. } \\
\text { Against chronic } \\
\text { bronchitis (in milk), } \\
\text { Migraine Therapy. }\end{array}$ & 83.5 & 92 & 0.111 & \\
\hline & $\begin{array}{l}\text { Solanum } \\
\text { lycopersicum L. }\end{array}$ & (LBNR232) & Tomates & Maticha & Fruits & Juice & Antianemic. & 100 & 86 & 0.104 & \\
\hline & $\begin{array}{l}\text { Solanum nigrum } \\
\text { L. }\end{array}$ & (LBNR233) & Morelle & Adil n'Ouchn & Leaves & $\begin{array}{l}\text { Decoction } \\
\text { Powder }\end{array}$ & $\begin{array}{l}\text { Antiulcer. } \\
\text { Promotes the } \\
\text { healing of wounds. }\end{array}$ & 73 & 28 & 0.033 & \\
\hline & $\begin{array}{l}\text { Solanum } \\
\text { tuberosum L. }\end{array}$ & (LBNR234) & Pomme de terre & Bttata & Tubers & $\begin{array}{l}\text { Poultice } \\
\text { Juice }\end{array}$ & $\begin{array}{l}\text { Antiseptic. } \\
\text { Stomachic }\end{array}$ & 84.8 & 58 & 0.07 & \\
\hline
\end{tabular}




\begin{tabular}{|c|c|c|c|c|c|c|c|c|c|c|c|}
\hline & $\begin{array}{l}\text { Withania } \\
\text { frutescens (L.) } \\
\text { Pauquy }\end{array}$ & (LBNR235) & Morelle & Tirnet & $\begin{array}{l}\text { Leaves } \\
\text { Roots }\end{array}$ & $\begin{array}{l}\text { Decoction } \\
\text { Juice } \\
\text { Infusion } \\
\text { Powder }\end{array}$ & $\begin{array}{l}\text { Heals asthma } \\
\text { Heals otitis. } \\
\text { Antidiabetic } \\
\text { Treat wounds, } \\
\text { abscesses and } \\
\text { smallpox. }\end{array}$ & 72.7 & 64 & 0.077 & \\
\hline Taxaceae & $\begin{array}{l}\text { Taxus baccata } \\
\text { L. }\end{array}$ & (LBNR236) & If commun & Igen & $\begin{array}{l}\text { Leaves } \\
\text { Roots }\end{array}$ & $\begin{array}{l}\text { Compress } \\
\text { Decoction }\end{array}$ & $\begin{array}{l}\text { Against the bites of } \\
\text { vipers. } \\
\text { Antidiabetic }\end{array}$ & 92 & 68 & 0.094 & 0.082 \\
\hline \multirow[t]{3}{*}{ Thymelaeaceae } & $\begin{array}{l}\text { Daphne gnidium } \\
\text { L. }\end{array}$ & (LBNR237) & Daphne garou & Lazzaz & Leaves & $\begin{array}{l}\text { Decoction } \\
\text { Infusion }\end{array}$ & $\begin{array}{l}\text { Heals toothache } \\
\text { Strengthens the hair } \\
\text { and stops their fall. }\end{array}$ & 72.2 & 63 & 0.076 & \multirow[t]{3}{*}{0.106} \\
\hline & $\begin{array}{l}\text { Thymelaea } \\
\text { hirsuta (L.) Endl }\end{array}$ & (LBNR238) & $\begin{array}{l}\text { Passerine } \\
\text { diöque }\end{array}$ & Mtnane & $\begin{array}{l}\text { Leaves } \\
\text { Stems }\end{array}$ & $\begin{array}{l}\text { Decoction } \\
\text { Infusion }\end{array}$ & $\begin{array}{l}\text { Against the fungi of } \\
\text { the feet. } \\
\text { Treatment of urinary } \\
\text { tract infection. } \\
\text { Antidiabetic. }\end{array}$ & 89 & 112 & 0.135 & \\
\hline & $\begin{array}{l}\text { Thymelea } \\
\text { virgata Mill. }\end{array}$ & (LBNR239) & Thymelée & Metnan & $\begin{array}{l}\text { Leaves } \\
\text { stems }\end{array}$ & Decoction & $\begin{array}{l}\text { Antidiabetic. } \\
\text { Diuretic. }\end{array}$ & 79.3 & 89 & 0.108 & \\
\hline \multirow[t]{2}{*}{ Urticaceae } & $\begin{array}{l}\text { Parietaria } \\
\text { mauritanica } \\
\text { Durieu }\end{array}$ & (LBNR240) & Pariétaire & Herrast lehjar & Leaves & $\begin{array}{l}\text { Poultice } \\
\text { Dcoction }\end{array}$ & $\begin{array}{l}\text { Anti-hemorrhoids. } \\
\text { Antiulcer. } \\
\text { Against nephritic } \\
\text { lithiasis }\end{array}$ & 96 & 121 & 0.146 & \multirow[t]{2}{*}{0.117} \\
\hline & Urtica urens L. & (LBNR241) & Ortie & Tikzint & $\begin{array}{l}\text { Flower } \\
\text { heads } \\
\text { Leaves }\end{array}$ & $\begin{array}{l}\text { Poultice } \\
\text { Infusion } \\
\text { Powder }\end{array}$ & $\begin{array}{l}\text { Against eczema and } \\
\text { hair loss. } \\
\text { Inflammation of the } \\
\text { urinary tract. } \\
\text { Antirheumatic. } \\
\text { Antidiabetic. }\end{array}$ & 66.9 & 73 & 0.088 & \\
\hline Verbenaceae & $\begin{array}{l}\text { Aloysia } \\
\text { citriodora Palau. }\end{array}$ & (LBNR242) & $\begin{array}{l}\text { Verveine } \\
\text { odorante }\end{array}$ & Lwizza & $\begin{array}{l}\text { Aerial } \\
\text { parts. }\end{array}$ & $\begin{array}{l}\text { Decoction } \\
\text { Infusion } \\
\text { Poultice }\end{array}$ & $\begin{array}{l}\text { Antidiabetic. } \\
\text { Calming. } \\
\text { Against intestinal } \\
\text { disorders in infants. }\end{array}$ & 73.6 & 79 & 0.095 & 0.095 \\
\hline Vitaceae & Vitis vinifera $L$. & (LBNR243) & Vigne rouge & Laanab & Leaves & $\begin{array}{l}\text { Poultice } \\
\text { Compress } \\
\text { Decoction }\end{array}$ & $\begin{array}{l}\text { Heals hemorrhoids. } \\
\text { Anti-migraine. } \\
\text { Promotes blood } \\
\text { circulation. } \\
\text { Antidiabetic. }\end{array}$ & 82.2 & 81 & 0.098 & 0.098 \\
\hline Xanthorrhoeaceae & $\begin{array}{l}\text { Aloe vera (L.) } \\
\text { Burm.f. }\end{array}$ & (LBNR244) & Aloès & Sibr & Leaves & Decoction & $\begin{array}{l}\text { Antidiabetic. } \\
\text { Anti Ulcer. } \\
\text { Sedative. }\end{array}$ & 100 & 101 & 0.122 & 0.105 \\
\hline
\end{tabular}




\begin{tabular}{|l|l|l|l|l|l|l|l|l|l|l|}
\hline & $\begin{array}{l}\text { Asphodelus } \\
\text { microcarpus } \\
\text { Salzm \& Viv. }\end{array}$ & (LBNR245) & $\begin{array}{l}\text { Asphodèle à } \\
\text { petite fruit. }\end{array}$ & Bliluz & $\begin{array}{l}\text { Roots } \\
\text { Leaves } \\
\text { Tubers }\end{array}$ & $\begin{array}{l}\text { Maceration } \\
\text { Decoction }\end{array}$ & $\begin{array}{l}\text { In olive oil to cure } \\
\text { the problems of the } \\
\text { ears. } \\
\text { Clean the } \\
\text { abscesses. } \\
\text { Antidiabetic. }\end{array}$ & 77.3 & 73 & 0.088 \\
\hline Zingiberaceae & $\begin{array}{l}\text { Alpinia } \\
\text { officinarum (L.) } \\
\text { Willd. }\end{array}$ & (LBNR246) & Le galanga & khoulandjan & Roots & $\begin{array}{l}\text { Decoction } \\
\text { Pntiemetic. } \\
\text { Anti-inflammatory. } \\
\text { Treat digestive } \\
\text { disorders } \\
\text { Aperitif. }\end{array}$ & 67 & 73 & 0.088 & 0.098 \\
\cline { 2 - 8 } & $\begin{array}{l}\text { Zingiber } \\
\text { officinale Rosc }\end{array}$ & (LBNR247) & Gingembre & Skinjbir & Roots & $\begin{array}{l}\text { Decoction } \\
\text { Poultice } \\
\text { Infusion }\end{array}$ & $\begin{array}{l}\text { Antidiabetic. } \\
\text { Heals rheumatic (in } \\
\text { olive oil). } \\
\text { Against the cold. }\end{array}$ & 83.5 & 89 & 0.108 \\
\hline Zygophyllaceae & $\begin{array}{l}\text { Zygophyllum } \\
\text { gaetulum } \\
\text { Emberger \& } \\
\text { Maire }\end{array}$ & (LBNR248) & Zygophylle & Aaggaya & Leaves & Infusion & $\begin{array}{l}\text { Antidiabetic. } \\
\text { Antiseptic } \\
\text { Antispasmodic } \\
\text { Anti-eczema. }\end{array}$ & 85.1 & 108 & 0.131 \\
\hline
\end{tabular}


The families are presented in alphabetical order. For every identified species is assigned: a scientific name, the common name, the used part, the method of preparation appropriate by the interviewees and the therapeutic use of these plants in the given area of study also the data of FIV, RFC, FC and FL.

Frequency of botanical familiesthe most used and their family use value (FIV):

Among the 70 families, the dominant families were Asteraceae (30 species), Lamiaceae (25 species each), followed by Apiaceae (18 species), Fabaceae (16 species), Poaceae and Rosaceae (12 species eachBrassicaceae and solanacae (9 species each),
Cucurbitaceae and Rutaceae (6 species each), the families of Cupressaceae, Euphorbiaceae, Malvaceae ,Myrtaceae, Oleaceae,and Renonculaceae were represented in the area by 4 species each, Amaranthaceae, Anacardiaceae, Aspargaceae, Caryophlaceae, Cistaceae, Papaveraceae, Plantaginaceae and Thymeleaceae were represented by 3 specieseach. These families alone represent 191 species or $77.02 \%$. The remaining 55 botanical families hold only one or two species each (57 species used or $22.98 \%$ ) (Fig .2).

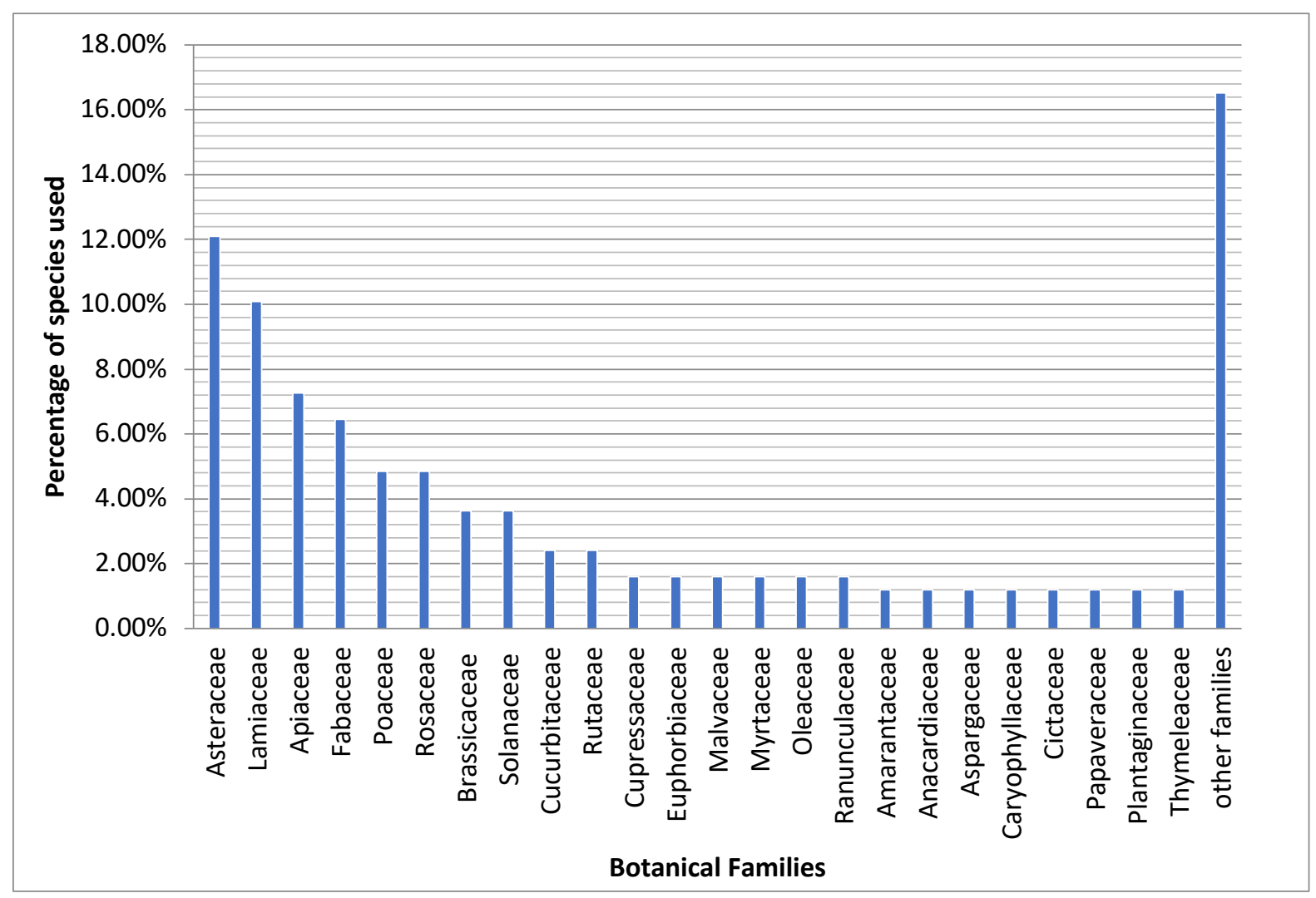

Figure 2. Percentage of species used (according to family).

Several ethnobotanical studies in Morocco revealed that Lamiaceae and Asteraceae were the most dominant (Mehdioui et al. 2007, Lahsissene et al. 2019).

Based on the FIV index, the10 most cited families were Euphorbiaceae (FIV=0.169), Myrtaceae $(F I V=0.165)$, Rhamnaceae ( $F I V=0.158)$, Rubiaceae $(\mathrm{FIV}=0.152)$, Ericaceae and Amaryllidaceae $(F I V=0.149), \quad$ Lamiaceae $(F I V=0.147)$, Iridaceae $(F I V=0.138), \quad$ Lyrthaceae $\quad(F I V=0.137) \quad$ and Ranunculaceae (FIV=0.133) (Fig. 3).

The abundance of these families may be explained by the geological nature in the study area, because the mountains offer a high availability of medicinal plants, which allow several species adapt easily to the climatic, geological and edaphic conditions from this region, for example Euphorbia resinifora (Euphorbiaceae), that exhibited a higher FIV (0.169) is at the same time an endemic species of the atlas of Azilal.

\section{RFC and FL plant species:}

The relative frequencies of citation (RFC) were used for evaluating the most used plant species by the interviewed. In this study RFC values ranged from 0.019 to 0.347 , and the most cited species by participants were Eucalyptus globulus Labill $(\mathrm{RFC}=0.347)$ and Euphorbia resinifera Berg $(\mathrm{RFC}=0.33$ ), because these plants were mentioned 
by a large number of informants, these species should be taken into account in future studies, to properly determine their efficiency, and they can also be used for phytochemical and pharmaceutical analysis to identify their active constituents for any drug extraction (Vitalini et al. 2013). On the other hand, the species having lowest values of RFC, such as, Buxus sempervirens L. $(\mathrm{RFC}=0.025)$ and Paronychia argentea Lam (RFC $=0.026)$, should not be abandoned, in order to be able to preserve the transcribed traces of phytotherapeutic practices, the transmission of which is essentially oral. This makes it a treasure that is diminishing over time in a society where orality is still a modus vivendi vivace (Table 2 ).

Fidelity level (FL), is an ethnopharmacological quantitative tools used to select for each disease which is the most effective medicinal plant, in the present study, FL values varied between $46 \%$ to $100 \%$. The calculation of the results showed that the most medicinal plants (140species), had a high fidelity value (>80\%), 33 species had a ( $=100 \%), 55$ medicinal plants had a FL $>60 \%$, and only the 18 remaining species show low fidelity values $(<60 \%)$ (Table2). These results show that the majority of the medicinal plants reported by the respondents have a high level of fidelity, that is to say a better healing potential, because they have been used to treat a single category of disease. In general, a FL of $100 \%$ for a specific plant indicates that all of the use-reports mentioned the same method for using the plant for treatment extraction (Srithi et al. 2009).

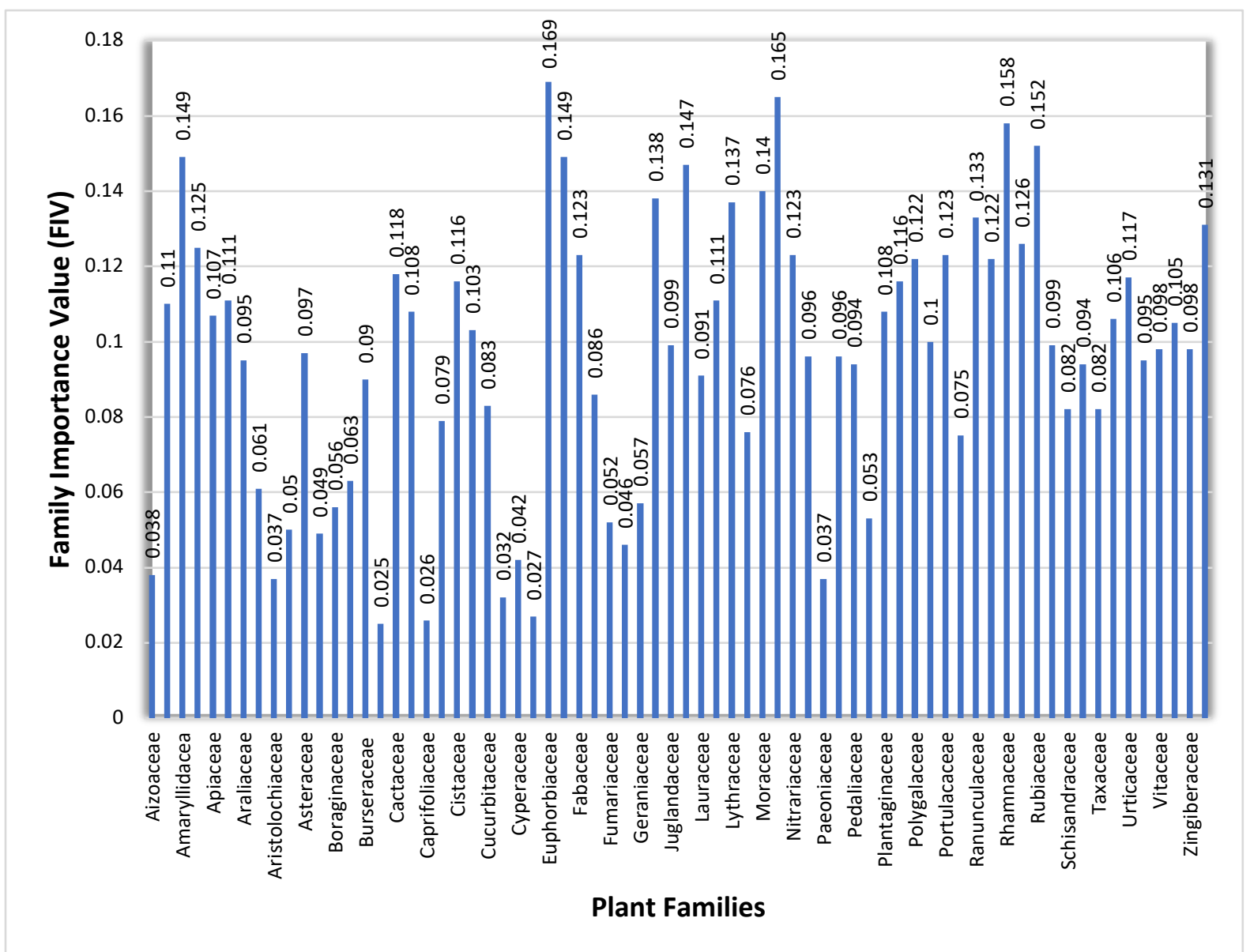

Figure 3 Family Importance Value (FIV) of medicinal plants.

\section{Parts of the medicinal plants used in the study area:}

The used parts of plants differed according to the plant itself, and the pathology treated. For example, the leaves of Pistacia lentiscus Desf are used for the treatment of gastric maldies, while its root is used to treat diarrhoea and the barks are antidiabetic. In contrast, only one part of the plant can be used for the treatment of different conditions, for example the roots of Cynaria humilis L. in powder are indicated for the treatment of the burns, and their decoction like anti-diabetic. The surveys carried out in the Central High Atlas make inferences to 16 parts: the leaves, the fruits, the seeds, the roots, the whole plant, the bark, the leafy stems, the aerial parts, the flowers, the flower heads, the stems, bulbs, young shoots, snowshoes, stigmas, nails. Results are illustrated in Fig 4. 
Leaves are the most used parts in traditional medicine recipes with a percentage of $21.25 \%$; also this high frequency of use of leaves can be interpreted by the ease and speed of their harvest (Bitsindou \& Lejoly 1996). Then come fruits and seeds with respective rates of $11.8 \%$ and $11.15 \%$. These two high percentages are probably related to the fact that these parts are accessible because they are apparent.

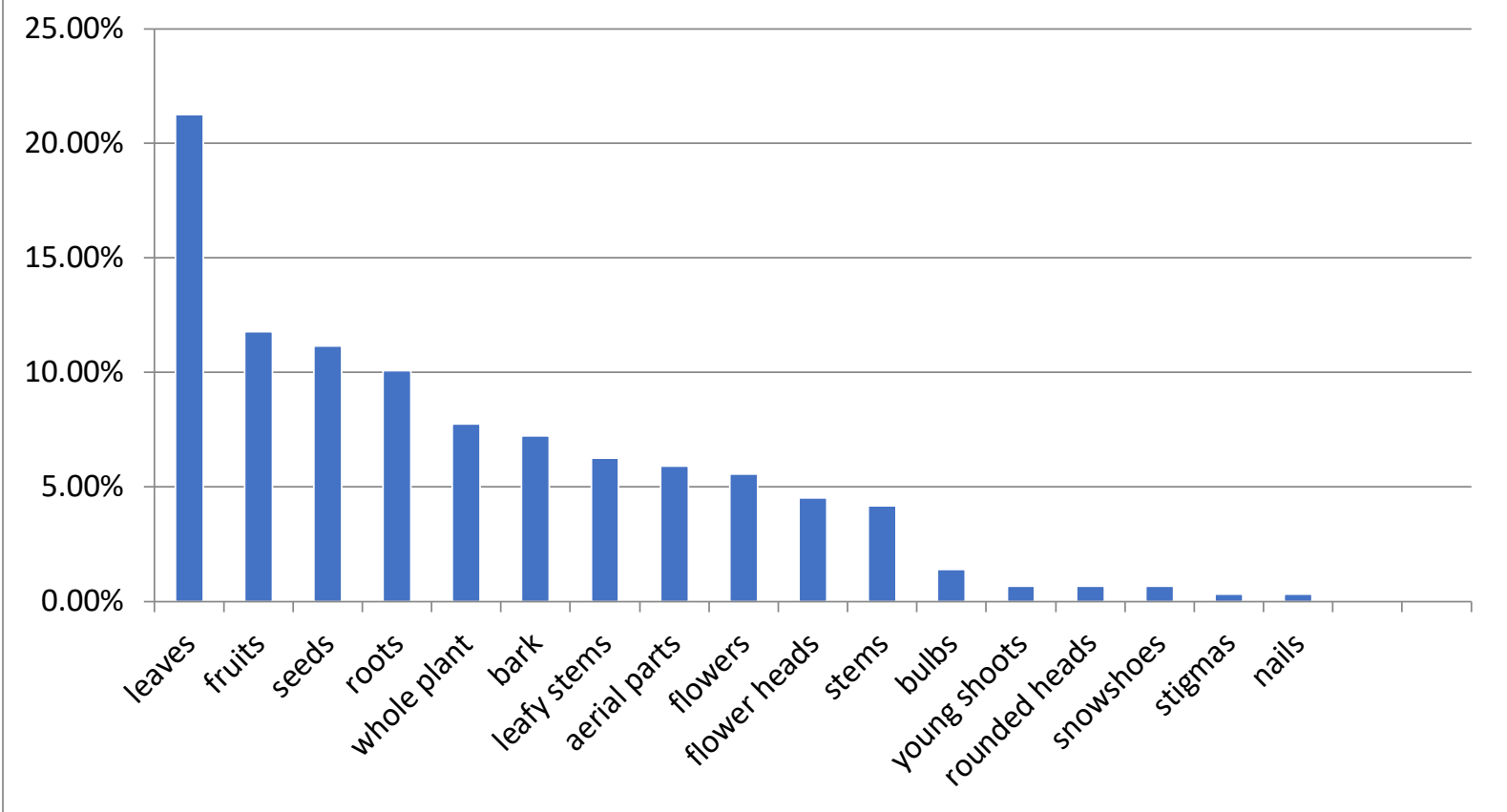

Figure 4. Percentage distribution of the different plant parts used

If taking the leaves as the most used organ of the plant into account, we noticed during our field prospections that the users remove the entire plant completely instead of looking only for the desired part (leaves). Since there is a clear relationship between the used part of the exploited plant and the effects of this exploitation on its being (Cunningham et al.1997). This collecting method seriously compromises the sustainability of the medicinal species concerned, especially the bulbous ones.

So, it is therefore in the leaves that photosynthesis takes place and sometimes constitutes the storage of secondary metabolites that are responsible for the biological properties of plants (Bigendako-polygenis \& Lejoly 1990), they are also characterized by their ease and rapid harvest (Bitsindou \& Lejoly 1996), and this may explain the high rate of foliage used by the population of the region.

Methods of preparation:

The plants harvested by the interviewee were prepared by the users themselves, on the other hand, the plants obtained at purchase were in prepared form (dried and sometimes crushed), so the same plant can be prepared differently, depending on the part used and the disease treated, for example: Eucalyptus globulus Labill leaves in decoction are antiseptic, and in poultice treat cough, flu and cold, while the bark decoction is antidiabetic. The methods of preparation most used and most feasible have been classified in descending order of magnitude: decoction (31.62\%), infusion (25.69\%), poultice $(14.58 \%)$, raw $(5.9 \%)$, maceration $(5.55 \%)$, cooked $(3.29 \%)$, compress $(2.61 \%)$, fumigation $(1.73 \%)$. The rest consists mainly of preparation methods rarely mentioned by the population and representing a rate of $9.03 \%$ (Fig. 5).

This shows that decoction was the most common method of preparation in the study area. Similar results are obtained in other studies conducted in Morocco (Chaachouay et al. 2019, El Rhaffari et al. 2002, Lahsissene et al. 2009, Mehdioui et al. 2007, Slimani et al. 2016).

It should also be noted that the majority of these preparations $(31.62 \%)$ are made by the herbal tea form (Fig. 6), because it is easily assimilated by the body, and it can collect the majority of active ingredients existing in medicinal plants, and by using the fresh parts of these plants (Fig. 7). Similar studies 
have shown that herbal tea is the most usable form of herbal remedies (El Alami et al. 2015, Hachi et al.
2015 Slimani et al. 2016), and that fresh parts are the most recommended (Abdurhman 2010).

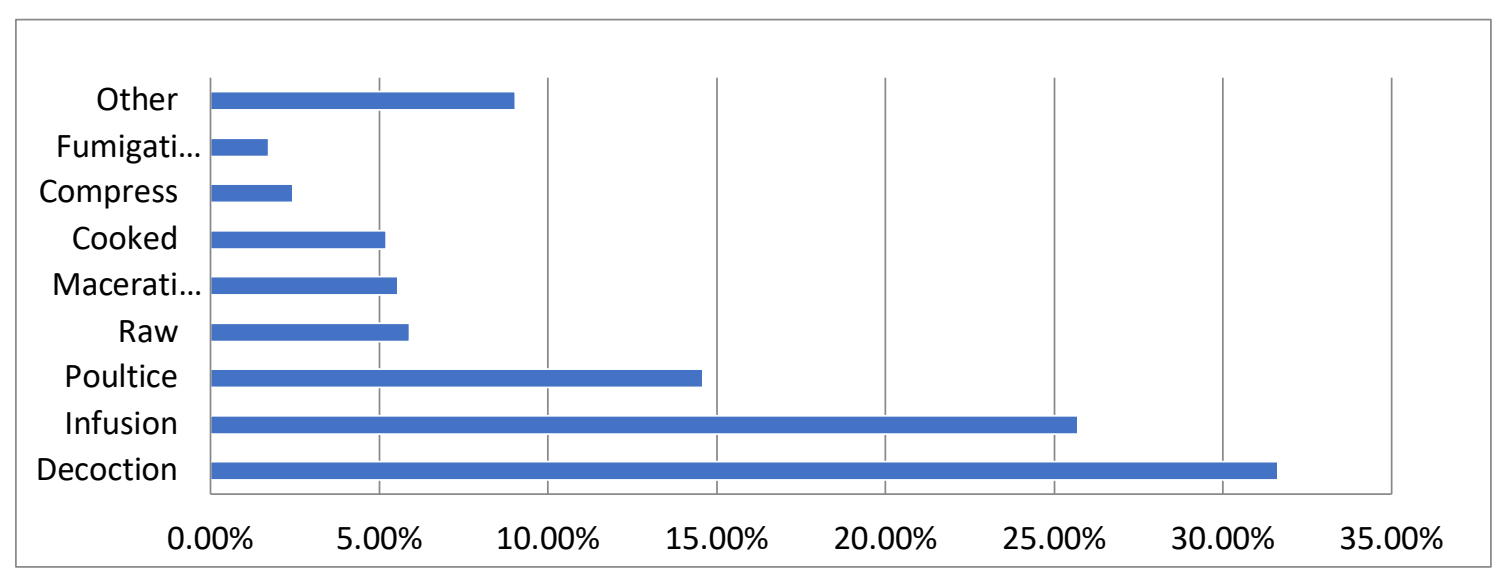

Figure 5. Percentage methods of preparation



Figure 6. Forms of preparation

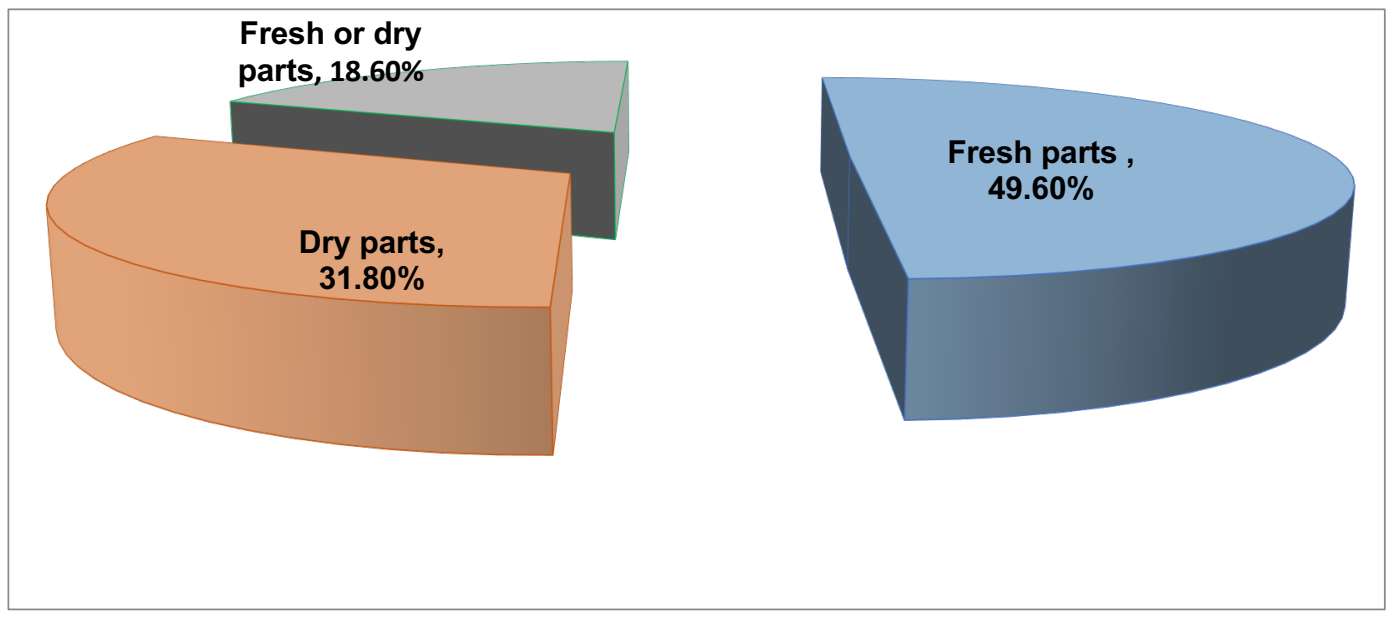

Figure 7. Percentage conditions of preparation

\section{Methods of Administration}

Several methods of employment were mentioned during our investigation, including the oral route, massage, brushing, gargling and rinsing were the main modes of employment, other modes of administration were less cited (Fig. 8). 
The respondents believed that the oral route is the most practical and safest way that facilitated the absorption of the active principles of medicinal plants, to transport them easily in the body, and consequently the cure of the target diseases. Similar findings indicated predominance of oral employment in Africa (Bousta et al. 2014, Cher-mat \& Gharzouli 2015, El Rhaffari et al. 2002, Rhattas et al. 2016).

\section{Distribution of medicinal plants according to the group of diseases treated}

The ethnopharmacological analysis has shown that the populations of the central High Atlas use the medicinal plants to treat different types of diseases. Nevertheless, it should be noted that a single plant can be used for the treatment of several ailments, and a single disease condition can be treated by several plants. For example, Artemisia herba-alba Asso is widely used in the study area, for its properties: antidiabetic, antispasmodic, anti septics, anthelmintics, and hypertensive and to treat urinary ailments. While dibetic diseases can be treated by several plants, for example, Euphorbia resinifera, Trigonella foenum, Salvia officinalis, Zygophyllum album. This study revealed that most of the plants represented in our region are used to treat gastrointestinal diseases(18.76\%), diabetic diseases (13.9\%), genitourinary infections (9.49\%), respiratory disorders (8.38\%) and skin diseases $(6.84 \%)$, while $5.29 \%$ of the species are used as antiseptic, $5.07 \%$ to treat rheumatological disorders, $4.63 \%$ for hair care, $4.42 \%$ to treat cardiovascular diseases and 3,53\% facial treatments. Some plants are used to stimulate appetite $(2.86 \%)$, others to treat neurological disorders $(2.65 \%)$ and to treat burns $(2.42 \%)$, Hepatic and haemostatic, ocular, auditory and anemic diseases are treated by less than $8 \%$ of the medicinal plants listed in the study area (Fig 9).Previous studies have shown that digestive diseases were the ailments most treated with medicinal plants (Daoudi et al. 2016, El Azzouzi \& Zidan 2015,Salhi et al. 2010, Tahri et al. 2012).

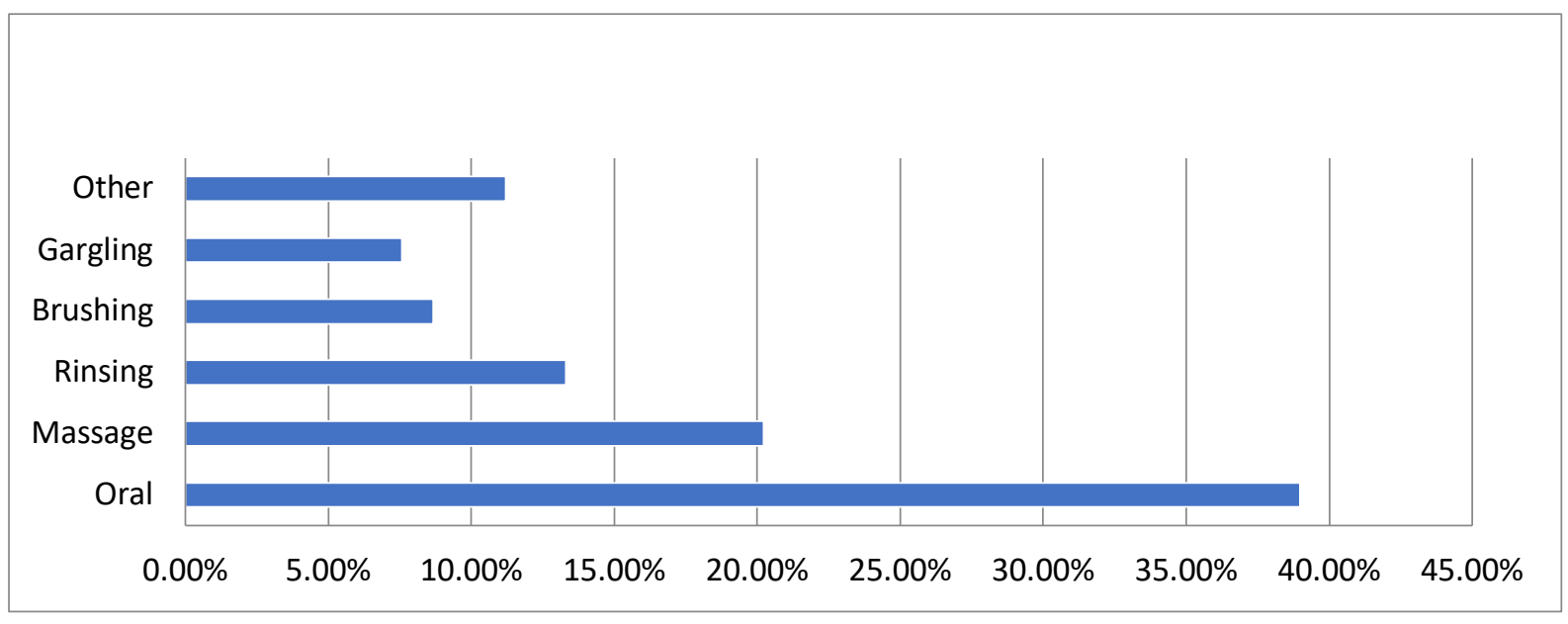

Figure 8. Percentage employment methods

\section{Traditional knowledge acquisition modes}

During our field survey, it was found that $39.81 \%$ of respondents based their choice of appropriate medicinal plants on the experience of their ancestors, who hold the traditional knowledge of treating diseases, while $32.14 \%$ consulted herbalists, $24.2 \%$ were referred on their own experience, because of the presence of many medicinal plants in their environment, or by reading books on traditional Arab medicine, or from television and radio programmes, while a small minority acquire their traditional knowledge through a doctor.This knowledge in phytotherapy was acquired by the local populations over centuries and is transmitted from one generation to another, which will safeguard ancestral knowledge. Especially since accumulated experience with age constitutes the main source of information at local level about the use of plants in traditional medicine (Fig. 10).

\section{The reasons for choosing this herbal medicine}

Herbal medicine in this study was used for its low cost in most patients (54\% of cases), and its effectiveness in $46 \%$ of cases. A study carried out in this area, in the Fès - Boulemane region in Morocco, had shown that phytotherapy is preferred by the local population for its low cost in most patients (54\% of cases), and its effectiveness in $38 \%$ of cases (Jouad et al 2001). 




Figure 9. Distribution percentage of medicinal plants uses depending on treated diseases group

\section{The results of care}

According to the ethnobotanical survey conducted in the Central High Atlas, we observed that $66.24 \%$ of people believed that medicinal plants help healing diseases, while $26.31 \%$ said that medicinal plants help only in improving health status, however, only $7.45 \%$ of the local population believed that medicinal plants can cause toxicities or may have side effects, if the user of these plants has not complied with the recommended dose and the technique of use (Fig. 11). Similar studies conducted in the Tafilalat region of south-eastern Morocco, indicated that $63 \%$ of people interviewed declared that modern medicine is the best (Eddouks et al. 2002). While in Guinea (in Black Africa), $85 \%$ of those participants were satisfied with the results of the use of medicinal plants (Baldé et al 2006). 


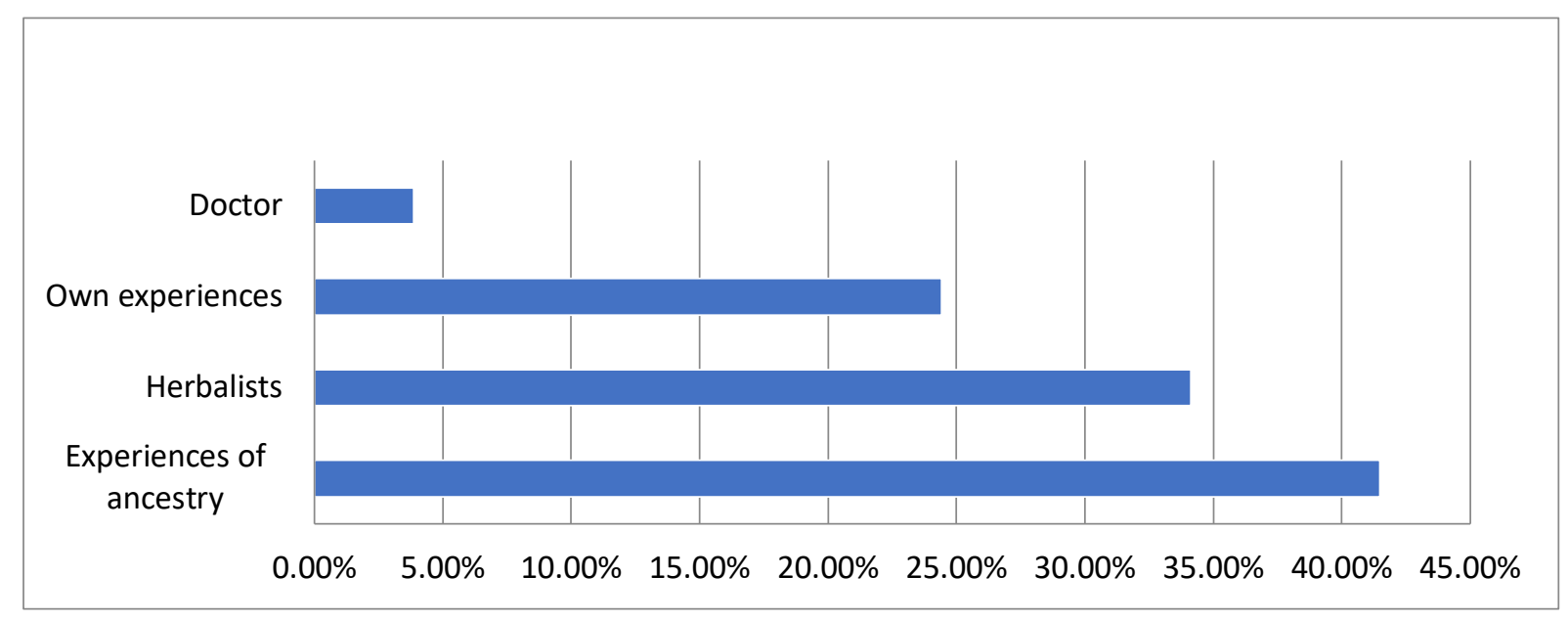

Figure 10. The reasons for choosing this herbal medicine

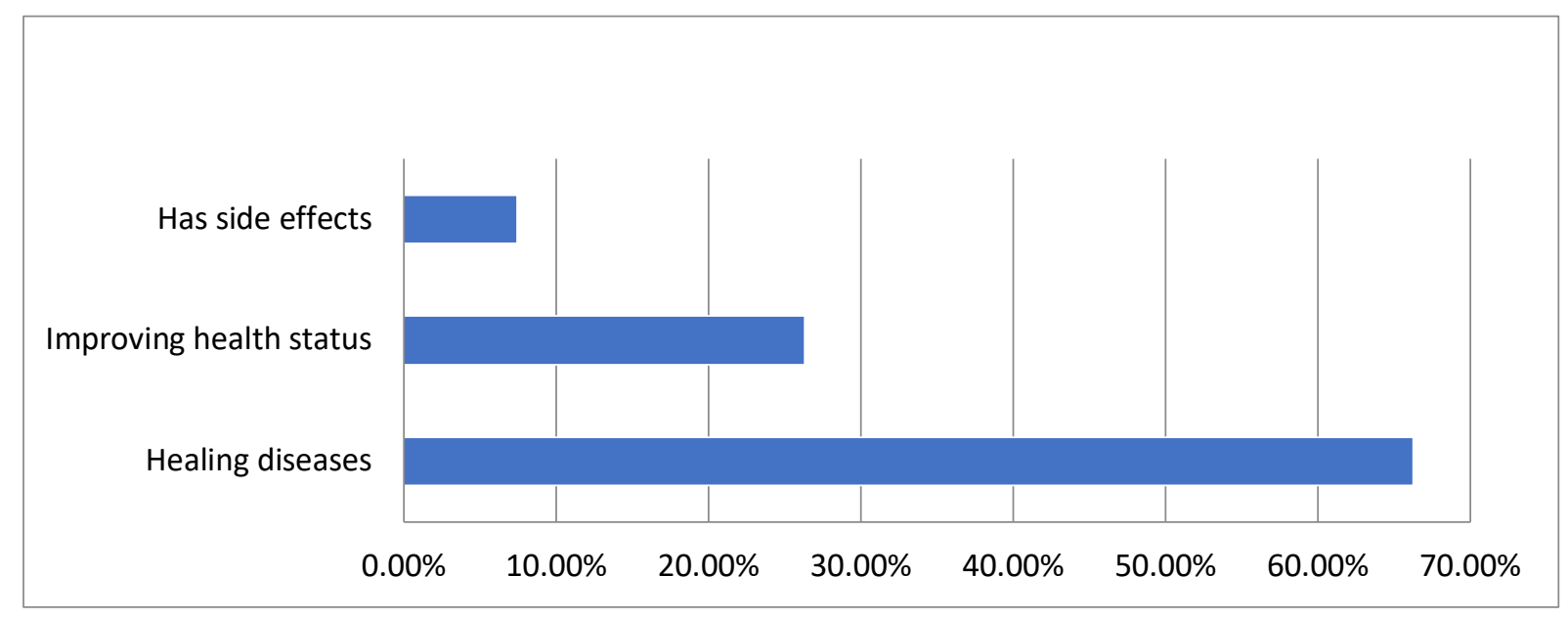

Figure 11. Results of care as a percentage

\section{Conclusions}

This study made it possible to determine the attachment of the population of the Central High Atlas of Morocco to the traditional heritage, and to collect information on the therapeutic uses practiced in this study area. Thus, the series of ethnobotanical and ethnopharmacological surveys revealed the region's wealth of medicinal plants, which constitute a very rich reservoir of biodiversity.

In this region, older women have a greater knowledge of phytotherapy and play an essential role in the conservation of therapeutic traditions based on medicinal plants and pass it on to their descendants.

From an ethnobotanical and pharmacological point of view, foliage is the most widely used part and decoction is the most widely used galenical form. Similarly, of all the diseases treated, gastrointestinal disorders and diabetes are the most frequently cited.
In addition, this study showed that the local populations of the Central High Atlas use 218 belonging to 73 families for the prevention and treatment of diseases. This region is the source of a very large number of medicinal plants for the whole of Morocco. This wealth of plants for therapeutic use is accompanied by the knowledge and practices of phytotherapy acquired by the inhabitants of the Atlas over the centuries.

Moreover, these results constitute a source of information that contributes to the knowledge of the medicinal flora and to the preservation of a local popular know-how that is tending to disappear. It will also be a database for the valorisation of medicinal plants in order to discover new active principles that can be used in pharmacology.

\section{Declarations}

List of abbreviations: Not applicable

Ethics approval and consent to participate: The study was approved by University Research Degree 
Committee of Kumaun University Nainital. All participants provided oral prior informed consent and signed in the questionnaire as their consent.

Consent for publication: Not applicable

Availability of data and materials: Not applicable Competing interests: The authors declare no competing interest.

Funding: The study did not receive any specific grant from funding agencies in the commercial, public or non-profit sectors.

Authors' contributions: SB: Carried out field survey in High Atlas Central, compiled the literature sources, analyzed the data collected, interpreted the results and wrote the manuscript. JD: Made a substantial contribution to data analysis and wrote the first draft of the manuscript. NB: provide help for data analysis and writing of the manuscript. LZ: Designed the research and identified the plant species. All the authors participated in writing and giving feedback on the manuscript and approved the final version of the manuscript.

\section{Acknowledgements}

We are grateful to the inhabitants and herbalists in thestudy area, who were cooperative during the ethnobotanical field surveys. Our sincere appreciation goes to all the people who helped us during the research work.

\section{Literature cited}

Aafi A, Ghanmi M, Satrani B, Aberchane M, Ismaili My R, EL Abid A. 2005. Diversité et valorisation des principales plantes aromatiques et médicinales (PAM) de l'écosystème cédraie au Maroc. Centre de Recherche Forestière B.P.763, Agdal-Rabat, Maroc.

Abdurhman N .2010. Ethnobotanical study of medicinal plants used by local people in Ofla Wereda, Southern Zone of Tigray: Region Ethiopia. Addis Ababa University: MSc Thesis.

Anyinam C. 1995. Exploring links between current environmental crisis and indigenous medical practices. Ecology and ethnomedicine 4:321-329.

Baldé NM, Youla A, Baldé MD, Kaké A, Diallo MM, Baldé MA, Maugendre D. 2006. Herbal medicine and treatment of diabetes in Africa: an example from Guinea.

Bammi J, Douira A. 2002. Les plantes médicinales dans la forêt de l'Achach (plateau central, Maroc.Acta Botanica Malacitana 27:131-145.

Bellakhdar J. 1997. La pharmacopée marocaine traditionnelle. Médecine arabe ancienne et savoirs populaires. Editions le Fennec, Casablanca/ Ibis Press. Paris, Pp. 760-764.
Bellakhdar J. 2006. Plantes médicinales et soins de base au Maghreb. Traité moderne de phytothérapieEd. Le Fennec, Casablanca, Maroc, 386 p. 145 planches couleurs.

Benlamdini N, Elhafian M, Rochdi A, ZidaneL. 2014. Étude floristique et ethnobotanique de la flore médicinale du Haut Atlas oriental (Haute Moulouya) (Maroc). Journal of Applied Biosciences 78 (1):67716787.

Bousta D, Boukhira S, Abderrahman A, Ghanmi M, el Mansouri L. 2014. Ethnopharmacological Study of anti-diabetic medicinal plants used in the MiddleAtlas region of Morocco (Sefrou region) .International Journal of Pharma Research and Health Sciences 2 (1):75-79.

Bigendako-Polygenis M, Lejoly J. 1990. La pharmacopée traditionnelle au Burundi, dans Ansay (N.) \& Thill (G.), éd., Pesticides et médicaments en santé animale. Rencontre Interdisciplinaire. Prélude Nord-Sud de Technologies. 1989/02. Bruxelles Liège. Namur: Presses Universitaires de Namur, Pp.425-442.

Bitsindou M, Lejoly J.1996. Les plantes antidiarrhéiques chez les Sundi de la souspréfecture de Kindamba (Congo). Laboratoire de Botanique Systematique et de Phytosociologie, Universite Libre de Bruxelles, Av. Paul Heger 28, CP 169, 1050 Bruxelles, Belgique.

Bulletin officiel $n^{\circ} 6340$ du 14 joumada I 1436 (5-32015), Décret $n^{\circ} 2-15-40$ du 1er joumada I 1436 (20 février 2015), 2015.

Chaachouay N, Benkhnigue O, El Ibaoui H, El Ayadi R, Zidane L. 2019.Medicinal plants used for diabetic problems in the Rif, Morocco. Ethnobotany Research \& Applications 18(21):1-29.

Chermat S, Gharzouli R .2015. Ethnobotanical study of medicinal flora in the North East of Algeria-An empirical knowledge in Djebel Zdimm (Setif), Journal of Materials Science and Engineering A 5: 50-59.

Cunningham $M$, Cunningham $A B$, Schippmann $U$. 1997. Trade in Prunus africana and the Implementation of CITES, German Federal Agency for Nature Conservation, Bonn, Germany.

Daoudi A, Bammou M, Zarkani S, Slimani I, Ibijbijen J, Nassiri L. 2016 .Etude ethnobotanique de la flore médicinale dans la commune rurale d'Aguelmouss province de Khénifra (Maroc). Phytothérapie 14(4):220-228.

Douiri E, El Hassani M, Bammi J, Badoc A, Douira A. 2007. Plantes vasculaires de la Moyenne Moulouya (Maroc oriental), Bulletin de la Société Linnéenne de Bordeaux. T. 142,142:409-438. 
Eddouks M, Maghrani M, Lemhadri A, Ouahidi L, Jouad H. 2002. Ethnopharmacological survey of medicinal plants used for the treatment of diabetes mellitus, hypertension and cardiac diseases in the south-east region of Morocco (Tafilalet). Journal of Ethnopharmacology, 82:97-103.

El Alami A, Farouk L, Chait A. 2016. Etude ethnobotanique sur les plantes médicinales spontanées poussant dans le versant nord de l'Atlas d'Azilal (Maroc), Algerian Journal of Natural 5 (12):71-282.

El Alami A, Chait A. 2017.Enquête ethnopharmacologique et ethnobotanique sur les plantes médicinales dans le Haut Atlas central du Maroc. Algerian Journal of Natural Products 4 (2):427-445.

El Azzouzi F, Zidane L. 2015. La flore médicinale traditionnelle de la région de Béni Mellal (Maroc). Journal of Applied Biosciences, 91:8493-8502.

El hafian M, Benlamdini N, Elyacoubi H, Zidane L. Rochdi A. 2014. Étude floristique et ethnobotanique des plantes médicinales utilisées au niveau de la préfecture d'Agadir-Ida -Outanane, Maroc. Journal of Applied Biosciences 81(1):7198-7213.

El Hilah FBA, Dahmani J, Belahbib N, Zidane L. 2015. Etude ethnobotanique des plantes médicinales utilisées dans le traitement des infections du système respiratoire dans le plateau central marocain. Journal of Animal \& Plant Sciences 25(2):3886-3897.

El Rhaffari L, Zaid A. 2002. Pratique de la phytothérapie dans le sud-est du Maroc (Tafilalet). Un savoir empirique pour une pharmacopée rénovée. Actes du 4ème congrès Européen d'Ethnopharmacologie : origine des pharmacopées traditionnelles et élaboration des pharmacopées savantes, Montpellier, France; Publiés par le CRD, Pp. 295-304.

El Yahyaoui O, Ait ouaaziz N, Sammama A, Kerrouri S, Bouabid B, Lrhorfi LA, Zidane L, Bengueddour R. 2015. Etude ethnobotanique: Plantes médicinales commercialisées à la province de Laâyoune; identification et utilisation 12(3):533541.

Emberger L, Maire M, Catalogue des plantes du Maroc ; Alger, Algérie 1941 ; vol 3, Pp.4-300.

Fennane M, Tattou MI, Mathez J, Quezel P. 1999. Flore pratique du Maroc: Manuel de determination des plantes vasculaires. Pteridophyta, Gymnospermae, Angiospermae (LauraceaeNeuradaceae). Institut scientifique.
Fennane M, Tattou MI. 2005. Flore vasculaire du Maroc Inventaire et Chorologie, Tome I. Trav. Inst. Sci. Rabat, série botanique, 37, 483 p.

Friedman J, Yaniv Z, Dafni A, Palewitch-A D. 1986. Preliminary classification of the healing potential of medicinal plants, based on a rational analysis of an ethnopharmacological field survey among Bedouins in the Negev Desert: Israel, J. Ethnopharmacol, $16: 275-287$.

Hachi M, Hachi T, Belahbib N, Dahmani J, Zidane L. 2015. Contribution a l'etude floristique et ethnobotanique de la flore medicinale utilisee au niveau de la ville de khenifra (Maroc). International Journal of Innovation and Applied Studies, 11(3):754.

HCP. 2018. Haut-commissariat au plan, Monograpie de la region Beni Mellal-Khenifra, Direction Regionale de Beni Mellal-Khenifra.

HCP. 2018. Haut-commissariat au plan, Monograpie de la region de Draa-Tafilalet, Direction Regionale de Draa-Tafilalet.

Hseini S, Kahouadji A. 2007. Étude ethnobotanique de la flore médicinale dans la région de Rabat, Maroc occidental. Lazaroa 28:79-93.

Idm'hand E, Msanda F, Cherifi K. 2019. Ethnopharmacological Documentation of Medicinal Plants Used in the

Traditional Treatment of Hypertension in Tarfaya Province, Morocco, International Journal of Pharmacology, Phytochemistry and Ethnomedicine 14:16-39.

Jain SK. 1964. The role of botanist in folklore research: Folklore, Ethnobotany of Bhil Tribe 5(4):145-150.

Jouad $H$, haloui $M$, Rhiouani $H .2001$. Ethnobotanical survey of medicinal plants used for the treatment of diabetes, cardiac and renal diseases in the north centre region of MOROCCO (fez -boulmane). Ethnophamacol, 77(2-3):175-82.

Kahouadji A. 1986. Recherches floristiques sur le massif montagneux des Béni Snassène (Maroc oriental), Thèse Magist., Univ. Sci. Tech. Du Languedoc, Montpellier, $235 \mathrm{p}$.

Laadim M, Ouahidi L, Zidane L, El Hessni A, Ouichou A, Mesfioui A. 2017.Ethnopharmacological survey of plants used for the treatment of diabetes in the town of Sidi Slimane(Morocco) Journal of Pharmacognosy and Phytotherapy, 9(6):101-110.

Lahsissene H, kahouadji A, Tijane M, Hseini S. 2009. Catalogue des plantes médicinales utilisées dans la région de Zaër (Maroc Occidental), Lejeunia, 186 p. 
Martin GJ. 2004. Ethnobotany: A Methods Manual, Earthscan Publ. Ltd, London, $01 \mathrm{p}$.

Mehdioui R, Kahouadji A. 2007. Etude ethnobotanique auprès de la population riveraine de la forêt d'Amsittène : cas de la Commune d'Imi n'Tlit (Province d'Essaouira). Bulletin de l'Institut Scientifique, 29:11-20.

Molares S, Ladio A. 2009. Chemosensory perception and medicinal plants for digestive ailments in a Mapuche community in NW Patagonia: Argentina. J. Ethnopharmacol. 123:397-406.

Ouchbani S, Romane F. 1995.Gradient climatique et répartition de la végétation dans l'Atlas de Béni Mellal (Maroc). Bulletin de l'Institut Scientifique, 19:53-64.

Pique A. 1994.Géologie du Maroc : les domaines régionaux et leur évolution structurale. Edit. PUMAG, Faculté des sciences Semlalia, Marrakech, Maroc, $119 \mathrm{p}$.

Quézel P, Santa S. 1962-1963. Nouvelle flore d'Algérie et des régions désertiques méridionales ; Tome 1, Tome 2, Editions du Centre National de la Recherche Scientifique, Paris, France.

Rhatta M, Douira A, Zidane L .2016. Etude ethnobotanique des plantes médicinales dans le Parc National de Talassemtane (Rif occidental du Maroc). Journal of Applied Biosciences, 97:9187 9211.

Rejdali M. 1996.La flore du Maroc : Etat actuel et perspectives de conservation. Diversité biologique et valorisation des plantes médicinales. Actes Edition:17-22.

Salhi S, Fadli M, Zidane L, Douira A. 2010.Etudes floristique et ethnobotanique des plantes médicinales de la ville de Kénitra (Maroc). Lazaroa, 31:133-146.

Sauvage CH, Vindt J. 1952. Flore du Maroc, analytique, descriptive et illustrée, Spermaphytes. Travaux de l'Institut Scientifique, Direction d'Instruction publique, Edit. Internationales : Tanger, Maroc, Facs.

Slimani I, Najem M, Belaidi R, Bachiri L, Bouiamrine EH, Nassiri L, Ibijbijen J. 2016. Etude ethnobotanique des plantes medicinales utilisees dans la region de Zerhoun-Maroc-[Ethnobotanical Survey of medicinal plants used in Zerhoun regionMorocco-]. International Journal of Innovation and Applied Studies 15(4) : 846.

Tahri N, El Basti A, ZidaneL, Rochdi A, Douira A. 2012.Etude Ethnobotanique Des Plantes Medicinales Dans La Province De Settat (Maroc).
Kastamonu Üni., Orman Fakültesi Dergisi 12 (2) :192-208.

Vijayakumar S, Yabesh JM, Prabhu S, Manikandan R, Muralidharan B. 2015. Quantitative ethnomedicinal study of plants used in the Nelliyampathy hills of Kerala, India. Journal of ethnopharmacology, 161:238-254.

Vitalini S, Iriti M, Puricelli C, Ciuchi D, Segale A, Fico G. 2013. Traditional knowledge on medicinal and food plants used in Val San Giacomo (Sondrio, Italy) -an alpine ethnobotanical study. Journal of Ethnopharmacology, 145(2), 517-529.

Ziyyat A, Legssyer A, Mekhfi H, Dassouli A, Serhrouchni M, Benjelloun W.1997. Phytotherapy of hypertension and diabetes in oriental Morocco, Journal of ethnopharmacology, 58(1):45-54.

Zougagh S, Belghiti A, Rochd T, Zerdani I, Mouslim J.2019. Medicinal and Aromatic Plants Used in Traditional Treatment of the Oral Pathology: The Ethnobotanical Survey in the Economic Capital Casablanca, Morocco (North Africa) Natural Products and Bioprospecting, 9:35-48 